\title{
SOLUTIONS TO THE OVERDETERMINED BOUNDARY PROBLEM FOR SEMILINEAR EQUATIONS WITH POSITION-DEPENDENT NONLINEARITIES
}

\author{
MIGUEL DOMÍNGUEZ-VÁZQUEZ, ALBERTO ENCISO, AND DANIEL PERALTA-SALAS
}

\begin{abstract}
We show that a wide range of overdetermined boundary problems for semilinear equations with position-dependent nonlinearities admits nontrivial solutions. The result holds true both on $\mathbb{R}^{n}$ and on compact Riemannian manifolds. As a byproduct of the proofs we also obtain some rigidity, or partial symmetry, results for solutions to overdetermined problems on Riemannian manifolds of nonconstant curvature.
\end{abstract}

\section{Introduction}

Let $M$ be a compact manifold of dimension $n \geqslant 2$ endowed with a Riemannian metric that we will denote by $g$ or $\langle\cdot, \cdot\rangle$. In this paper we are interested in proving the existence of domains $\Omega \subset M$ where there is a nontrivial solution $u>0$ to the overdetermined boundary value problem

$$
\left\{\begin{aligned}
\Delta_{g} u+\lambda f(\cdot, u) & =0 & & \text { in } \Omega \\
u & =0 & & \text { on } \partial \Omega \\
\left\langle\nabla_{g} u, \nu_{g}\right\rangle & =\text { constant } & & \text { on } \partial \Omega .
\end{aligned}\right.
$$

Here $\lambda$ is some real constant, $f(p, z) \in C_{\text {loc }}^{1, \alpha}(M \times \mathbb{R})$ is a given nonlinearity that may depend on the point $p$ of the manifold $M, \Delta_{g}$ is the Laplacian on the manifold and, as is customary, the Neumann condition is formulated in terms of the gradient with respect to the metric $g$, which we denote by $\nabla_{g}$, and the unit outer normal $\nu_{g}$ of the domain.

It should be emphasized that essentially all the existing literature on overdetermined problems focuses on the case when the nonlinearity only depends on $u$, that is, $f(p, z)=G(z)$ for some function $G \in C_{\mathrm{loc}}^{1, \alpha}(\mathbb{R})$, in which case the domains for which the overdetermined problem admits a solution are usually called $G$-extremal domains:

$$
\left\{\begin{aligned}
\Delta_{g} u+\lambda G(u) & =0 & & \text { in } \Omega \\
u & =0 & & \text { on } \partial \Omega \\
\left\langle\nabla_{g} u, \nu_{g}\right\rangle & =\text { constant } & & \text { on } \partial \Omega .
\end{aligned}\right.
$$

The investigation of overdetermined boundary value problems traces back to Serrin's seminal paper [36] in 1971, which concerns translation-invariant overdetermined problems on $\mathbb{R}^{n}$ of the form (1.2), the model case being $G(z)=1$. In this case, it is clear that there is a radial solution to the overdetermined problem

2010 Mathematics Subject Classification. 35N25, 49Q10, 58J05, 58J32, 58J37.

Key words and phrases. Overdetermined boundary value problems, semilinear elliptic problems, asymptotically homogeneous spaces, symmetric spaces, harmonic spaces. 
when the domain is a ball. By generalizing the moving plane method developed by Alexandrov [1] in 1956 to study constant mean curvature hypersurfaces in $\mathbb{R}^{n}$, Serrin was able to show that, in a way, those are the only solutions to the overdetermined problem on bounded domains. In other words, under mild assumptions it is known that if (1.2) admits a solution for a bounded domain $\Omega$ of $\mathbb{R}^{n}$, then $\Omega$ must be a Euclidean ball and the solution $u$ is radial. This method works also for the hyperbolic space and hemisphere 26 due to the existence of many totally geodesic hypersurfaces, but fails for more general geometries of nonconstant curvature. Other important symmetry or classification results concerning (mainly unbounded) $G$-extremal domains in spaces of constant curvature can be found in [6, 11, 12, 13, 32, 39.

Existence or symmetry results for overdetermined problems in Riemannian manifolds of nonconstant curvature are very scarce. Nontrivial existence results of $G$ extremal domains in flat spaces can be found in 30, 33, 34, 37, and also for some specific manifolds in 28] and [10. The latter also provides a symmetry result on certain manifolds. In fact, in general Riemannian manifolds, the very strong requirement that constant Dirichlet and Neumann conditions be satisfied simultaneously may even lead one to conjecture that such solutions should not exist for generic metrics. However, in two surprising papers, Pacard and Sicbaldi 31 and Delay and Sicbaldi [9] proved the existence of extremal domains with small volume for the first eigenvalue $\lambda_{1}$ of the Laplacian in any compact Riemannian manifold, so Problem (1.2) admits solutions with $G(z):=z$ and $\lambda:=\lambda_{1}(\Omega)$ for any compact $M$. A similar approach was followed by Fall and Minlend [15] to show the existence of solutions to (1.2) when $G$ is constant.

Our first objective in this paper is to provide existence results for the nonlinear overdetermined problem (1.1) for a wide range of nonlinearities $f(p, z)$, possibly depending on the point $p$ in the manifold. The solution domains $\Omega$ that we construct to the Problem (1.1) are perturbations of small geodesic balls centered at suitably chosen points of the manifold. One can state the result as follows, where $B_{r}$ denotes the ball in $\mathbb{R}^{n}$ of radius $r,\left|B_{r}\right|$ is its volume, and $f_{z}, f_{z z}$ denote the first and second partial derivatives of $f(p, z)$ with respect to $z$. Notice that in the hypotheses we do not impose any restrictions on the growth of $f(p, z)$ as $z \rightarrow \infty$ and that there are no global sign conditions.

Theorem 1.1. Let $M$ be a compact Riemannian manifold, and $f(p, z)$ any function that satisfies one of the following two conditions:

(i) $f \in C_{\mathrm{loc}}^{1, \alpha}(M \times \mathbb{R})$ and $f(p, 0)>0$ for all $p \in M$.

(ii) $f \in C_{\mathrm{loc}}^{2}(M \times \mathbb{R}), f(p, 0)=0, f_{z}(p, 0)=c>0$ and $f_{z z}(p, 0) \neq 0$ for all $p \in M$, where $c$ is a constant independent of $p$.

Then for every small enough positive $\varepsilon$ there exists a domain $\Omega \subset M$ with volume equal to $\left|B_{\varepsilon}\right|$ and a positive constant $\lambda$, which is of order $\varepsilon^{-2}$, such that the overdetermined problem (1.1) admits a positive solution. The domain $\Omega$ is a $C^{2, \alpha}$-small perturbation of a geodesic ball of radius $\varepsilon$.

The construction of solutions to (1.1) builds upon ideas of Pacard-Sicbaldi 31] and Delay-Sicbaldi [9] in the case of linear, position-independent equations, and extends them to the case of semilinear equations with position-dependent nonlinearities. The strategy is as follows. Firstly, we carry out an analysis of the nonlinearities considered in the statement of Theorem 1.1 and, using some bifurcation 
arguments, we prove that they satisfy certain rather nontrivial technical conditions that are crucially employed in several steps of the demonstration. Next we show that a domain $\Omega$ that is a small perturbation of a small geodesic ball in $M$ admits a positive solution $u \in C^{2, \alpha}(\Omega)$ to $\Delta_{g} u+\varepsilon^{-2} f(\cdot, u)=0$ in $\Omega,\left.u\right|_{\partial \Omega}=0$, where $\varepsilon>0$ is somehow related to the size of $\Omega$. Then we consider an operator $\mathcal{F}$ that measures how far the normal derivative of $u$ along $\partial \Omega$ is from being constant. We calculate the linearization of $\mathcal{F}$, and study some properties of the linearized operator. This allows us to apply the implicit function theorem in Banach spaces to $\mathcal{F}$ and prove that, for any $p \in M$ and for any small enough $\varepsilon>0$, there exists a small perturbation $\Omega_{\varepsilon, p}$ of a geodesic ball centered at $p$ that admits a solution $u_{\varepsilon, p}$ satisfying not only the equation and the Dirichlet condition, but also the Neumann condition up to, roughly speaking, a linear error. This linear error is controlled by certain vector field $a_{\varepsilon, p}$ on $M$. Then we consider, for each small enough $\varepsilon>0$, a smooth map $\mathcal{J}_{\varepsilon}: M \rightarrow \mathbb{R}$ that associates to each point $p$ the energy of $u_{\varepsilon, p}$, that is,

$$
\mathcal{J}_{\varepsilon}(p):=\int_{\Omega_{\varepsilon, p}}\left(\frac{1}{2}\left|\nabla_{g} u_{\varepsilon, p}\right|_{g}^{2}-F\left(\cdot, u_{\varepsilon, p}\right)\right) d V_{g}
$$

where $F(p, z):=\int_{0}^{z} f(p, \zeta) d \zeta$ and $d V_{g}$ is the Riemannian measure induced by $g$. The critical points of the function $\mathcal{J}_{\varepsilon}$ are precisely the zeros of the vector field $a_{\varepsilon, p}$. Finally, it is shown that $u_{\varepsilon, p}$ satisfies the Neumann condition precisely if $p$ is a critical point of $\mathcal{J}_{\varepsilon}$. If $M$ is compact, then $\mathcal{J}_{\varepsilon}$ has a critical point; indeed, it has at least as many critical points as the Lusternik-Shnirelmann category of $M$ [7]. Therefore, for every small enough $\varepsilon>0$ we obtain domains $\Omega$, with volume $\left|B_{\varepsilon}\right|$ and enclosing certain specific points $p \in M$, where the overdetermined problem with constant of order $\varepsilon^{-2}$ admits a nontrivial solution. The points of the manifold at which one centers the solution domains turn out to be determined by the zeros of the vector field $a_{\varepsilon, p}$.

Notice that, as the zeros of a vector field are ultimately involved, it is not hard to believe that when the Euler characteristic of the manifold is nonzero the result holds in greater generality. This idea leads to the following slightly stronger result, which allows for the presence of first order terms. We have chosen to state it in the Introduction because, for this reason, it applies to problems that do not have a variational structure:

Theorem 1.2. Suppose that the Euler characteristic of the compact manifold $M$ is nonzero and let $X$ be a vector field on $M$ of class $C^{0, \alpha}$. Then the statements of Theorem 1.1 remain true for the more general equation

$$
\Delta_{g} u+\left\langle\nabla_{g} u, X\right\rangle+\lambda f(\cdot, u)=0 \quad \text { in } \Omega
$$

with the same assumptions on the nonlinearity $f$ and the same overdetermined boundary conditions:

$$
u=0 \quad \text { and } \quad\left\langle\nabla_{g} u, \nu\right\rangle=\text { constant } \quad \text { on } \partial \Omega .
$$

It is worth stressing that Theorem 1.1 provides new existence results even for overdetermined problems with the flat Laplacian on $\mathbb{R}^{n}$; in particular, it automatically ensures the existence of a positive solution for the overdetermined problem

$$
\left\{\begin{aligned}
\Delta u+\lambda f(\cdot, u) & =0 & & \text { in } \Omega \\
u & =0 & & \text { on } \partial \Omega \\
\partial_{\nu} u & =\text { constant } & & \text { on } \partial \Omega
\end{aligned}\right.
$$


where $\Omega \subset \mathbb{R}^{n}$ and $\Delta$ denotes the standard Laplacian, for any nonlinearity $f(x, z)$ that is periodic in $x$ and satisfies Condition (i) or (ii) in Theorem 1.1. This is clear because one can reformulate the problem on a flat $n$-dimensional torus, which falls within the scope of Theorem 1.1 .

Perhaps more interestingly, one can obtain existence results on $\mathbb{R}^{n}$ even for nonlinearities that are non-periodic in $x$. This is because the method of proof of Theorem 1.1 can be extended to manifolds that are not necessarily compact but have some asymptotic symmetry properties that one could call "asymptotic homogeneity". Details are provided in Section 5. To illustrate this fact we will next present a very particular instance of Theorem 5.6. which is stated and proved in Section 5

Theorem 1.3. Assume that one of the following two conditions holds:

(i) $f(x, z) \in C_{\mathrm{loc}}^{1, \alpha}\left(\mathbb{R}^{n} \times \mathbb{R}\right)$ converges to $G(z) \in C_{\mathrm{loc}}^{1, \alpha}(\mathbb{R})$ locally uniformly in $C^{1, \alpha}$ as $|x| \rightarrow \infty$ and $\inf _{x \in \mathbb{R}^{n}} f(x, 0)>0$.

(ii) $f(x, z) \in C_{\text {loc }}^{2}\left(\mathbb{R}^{n} \times \mathbb{R}\right)$ converges to $G(z) \in C_{\mathrm{loc}}^{2}(\mathbb{R})$ locally uniformly in $C^{2}$ as $|x| \rightarrow \infty, f(x, 0)=0, f_{z}(x, 0)=c$ and $\left|f_{z z}(x, 0)\right|>c^{\prime}$ for all $x \in \mathbb{R}^{n}$, where $c, c^{\prime}$ are positive constants independent of $x$.

Then for every small enough $\varepsilon$ there exists a domain $\Omega \subset \mathbb{R}^{n}$, which is a $C^{2, \alpha}$-small deformation of a ball of radius $\varepsilon$ with the same volume, and a positive constant $\lambda$ of order $\varepsilon^{-2}$ such that the overdetermined problem

$$
\left\{\begin{aligned}
\Delta u+\lambda f(\cdot, u) & =0 & & \text { in } \Omega \\
u & =0 & & \text { on } \partial \Omega \\
\partial_{\nu} u & =\text { constant } & & \text { on } \partial \Omega
\end{aligned}\right.
$$

admits a positive solution.

When the nonlinearity $f(p, z)=G(z)$ is independent of $p$, the arguments involved in the proof of Theorem1.1 yield as a byproduct a uniqueness result that can be used to prove the symmetry of certain $G$-extremal domains. With the exception of [10, the existing symmetry results for overdetermined problems only apply when $M$ is a space of constant curvature, that is, a Euclidean space, a hyperbolic space, a round sphere, or a quotient thereof. The intuitive reason of this is that these symmetry results are typically obtained using the moving plane method, so in a certain sense they hinge on the idea of using isometries (that is, rigid motions) to transport planes. A plane is determined by three points, and the key property of spaces of constant curvature that is ultimately employed is that they are the only three-point homogeneous spaces, which means that given two triples of points $\left(p_{1}, p_{2}, p_{3}\right)$ and $\left(p_{1}^{\prime}, p_{2}^{\prime}, p_{3}^{\prime}\right)$ on the manifold with the same relative distances (i.e., $\left.d\left(p_{i}, p_{j}\right)=d\left(p_{i}^{\prime}, p_{j}^{\prime}\right)\right)$, then there is an isometry $\varphi$ such that $\varphi\left(p_{j}\right)=p_{j}^{\prime}$.

Our symmetry results apply to Riemannian manifolds that also have a large isometry group, but not large enough to be three-point homogeneous, which means that the moving plane method will not work. Specifically, we prove symmetry results in harmonic spaces, which are defined as those Riemannian manifolds whose geodesic spheres of sufficiently small radius have constant mean curvature. Examples of harmonic spaces are the isotropic homogeneous spaces (also referred to as two-point homogeneous spaces), such as the projective and hyperbolic spaces over the distinct division algebras (that is, the complex numbers, the quaternions and 
the octonions). We refer to Section 6 for more information on these spaces. As we will see, the price to pay for this greater generality is that one cannot prove the symmetry of any $G$-extremal domain, but only of those that are close enough to being a small geodesic ball. Therefore, this is a rigidity result for domains close to small geodesic spheres where the overdetermined problem admits a nontrivial solution.

In order to state this kind of results, it is convenient to introduce some notation. First, given a closed hypersurface $\Sigma$ in $M$, we define its center of mass as the minimum of the function

$$
p \in M \mapsto \frac{1}{2} \int_{\Sigma} d^{2}(p, q) d \sigma_{g}
$$

whenever it exists and is unique, where $d$ is the Riemannian distance on $M$ and $d \sigma_{g}$ is the induced Riemannian volume form on $\Sigma$. With a slight abuse of notation, we will say that a domain $\Omega$ is centered at $p$ if the center of mass of its border $\partial \Omega$ is $p$. We refer e.g. to [23, 29] for details. Second, given a point $p \in M$ and a continuous positive function $h$ defined on the unit tangent sphere $T_{p}^{1} M$ at $p$ that is not too large in $L^{\infty}$-norm (specifically, smaller than the injectivity radius at $p$ ), we find it convenient to introduce the notation

$$
B_{h}^{g}(p):=\left\{\exp _{p}^{g}(x): x \in T_{p} M, 0 \leqslant|x|<h(x /|x|)\right\},
$$

where $\exp ^{g}$ denotes the exponential map of $(M, g)$. It is clear that if $\Omega$ is a domain that is close enough to a geodesic ball centered at $p$, in a sense that can be made precise easily, then there is some function $h$ as above such that $\Omega=B_{h}^{g}(p)$.

We are now ready to state an important special case of our symmetry results, which asserts that any $G$-extremal domain in a harmonic space that is close enough to a small geodesic ball is indeed a geodesic ball if the nonlinearity is, roughly speaking, concave and sublinear:

Theorem 1.4. Let $M$ be a harmonic space of dimension $n \geqslant 2$, and let $G \in$ $C_{\text {loc }}^{1, \alpha}(\mathbb{R})$ be concave, with $G(0)>0$, and such that either $G\left(z_{0}\right)=0$ for some $z_{0}>0$ or $\lim _{z \rightarrow+\infty} \frac{G(z)}{z}=0$. Suppose that $\Omega=B_{\varepsilon(1+v)}^{g}(p)$ is a G-extremal domain centered at a certain point $p$, i.e., that there is a nontrivial solution to the overdetermined problem (1.2) in this domain. If $\varepsilon, \lambda \varepsilon^{2}$ and $\|v\|_{C^{2, \alpha}\left(T_{p}^{1} M\right)}$ are small enough, then $\Omega$ is a geodesic ball and $u$ only depends on the geodesic distance to $p$.

This result applies, for instance, to the model overdetermined problem $G(z)=1$ on a harmonic space, showing that any domain close to a small enough geodesic ball where the corresponding overdetermined problem admits a solution is exactly a geodesic ball. The same symmetry result turns out to hold for the extremal domains associated with the first eigenvalue problem, that is, Problem (1.2) with $\lambda G(z)=\lambda_{1}(\Omega) z($ see Remark 6.6) $)$.

This paper is organized as follows. In Section 2 we state a technical hypothesis that we call Assumption A and give sufficient conditions ensuring that it is satisfied, which correspond to conditions (i)-(ii) in Theorem 1.1. In Section 3 we prove Theorem 1.1 using that the nonlinearities we consider satisfy Assumption A. This involves solving the Dirichlet problem for small perturbations of small geodesic balls and studying the operator that encodes the failure of the Neumann condition. Then, 
we use a geometric variational approach to show that the solutions to (1.1) correspond to the critical points of the functions $\mathcal{J}_{\varepsilon}$, which are analyzed subsequently. In Section 4 we consider variations on the proof of Theorem 1.1 proving, in particular, Theorem 1.2. In Section 5 we discuss the possibility of relaxing the compactness assumption on $M$ and show the validity of our existence results under different approximate homogeneity hypotheses, establishing Theorem 1.3 as a particular case. Finally, Section 6] is devoted to several symmetry results for $G$-extremal domains in specific Riemannian manifolds, such as two-point homogeneous, symmetric or harmonic spaces, including Theorem 1.4.

\section{Analysis of the nONLINEARITIES CONSIDERED IN TheOREM 1.1}

In this section we show that the nonlinearities considered in Theorem 1.1 satisfy certain technical properties that will be extensively used in the paper and which we call Assumption A. To state the assumptions, we will henceforth use the notations $\nabla$ and $\Delta$ for the Euclidean gradient and Laplacian, respectively. Throughout we are assuming that the function $f$ is of class $C_{\text {loc }}^{1, \alpha}(M \times \mathbb{R})$.

Roughly speaking, Assumption A asserts that, when the point on the manifold is "frozen", the nonlinearity is such that there are positive solutions to the associated Dirichlet problem in the unit ball of $\mathbb{R}^{n}$, and that a linear partial differential operator and countably many linear ODEs defined in terms of this function satisfy certain nondegeneracy conditions. Specifically, Assumption A can be stated as follows:

Assumption A. For each point $p \in M$ there exists a radial function $\phi_{p}(x)$ of class $C^{2, \alpha}$ on the unit ball, varying differentiably with $p$, and a constant $\bar{\lambda}>0$ independent of $p$ such that:

(i) $\phi_{p}(x)$ is positive in $B_{1}$ and solves the Dirichlet problem

$$
\left\{\begin{array}{rlll}
\Delta_{x} \phi_{p}(x)+\bar{\lambda} f\left(p, \phi_{p}(x)\right) & =0 & \text { in } B_{1}, \\
\phi_{p} & = & 0 & \text { on } \partial B_{1},
\end{array}\right.
$$

with $\left.\partial_{\nu} \phi_{p}\right|_{\partial B_{1}}<0$.

(ii) The linear operator $\Delta+\bar{\lambda} f_{z}\left(p, \phi_{p}\right): C_{\text {Dir }}^{2, \alpha}\left(B_{1}\right) \rightarrow C^{0, \alpha}\left(B_{1}\right)$ is invertible, where $C_{\text {Dir }}^{2, \alpha}\left(B_{1}\right)$ denotes the subspace of functions of $C^{2, \alpha}\left(B_{1}\right)$ that vanish on $\partial B_{1}$.

(iii) For each nonnegative integer $j$, let $a_{p, j}(r)$ be the only solution to the equation

$$
\begin{aligned}
a_{p, j}^{\prime \prime}(r)+\frac{n-1}{r} a_{p, j}^{\prime}(r)+\left(\bar{\lambda} f_{z}\left(p, \phi_{p}(r)\right)-\right. & \left.\frac{j(j+n-2)}{r^{2}}\right) a_{p, j}(r) \\
& =\frac{n-1-j(j+n-2)}{r^{2}} \partial_{r} \phi_{p}(r)
\end{aligned}
$$

with initial conditions $a_{p, j}(1)=a_{p, j}^{\prime}(1)=0$. Here, since $\phi_{p}(x)$ is a radial function, we are writing $\phi_{p}(r)$ with the obvious meaning. Then

$$
\lim _{r \rightarrow 0^{+}} a_{p, j}(r) \neq 0
$$

for all $j \geqslant 2$. 
Remark 2.1. The characteristic exponents of the ODE (2.1) at 0 are $-j$ and $n+j-2$, so the meaning of condition (iii) is that, for all $j \geqslant 2$ and $p \in M, r=1$ is not a double zero of the solution to the only solution to the ODE (2.1) that is continuous at $r=0$.

Remark 2.2. Using the constant $\lambda$ that appears in the overdetermined problem (1.1), without any loss of generality we take the constant $\bar{\lambda}$ appearing in Assumption $\mathrm{A}$ to be $\bar{\lambda}=1$, after multiplying the function $f$ by a constant if necessary. We will do this without any further mention when we use Assumption A in subsequent sections.

In the following theorem we provide a wide class of nonlinearities that satisfy Assumption A, corresponding to the first class of examples provided in the Introduction:

Theorem 2.3. Suppose that $M$ is compact and $f(p, 0)>0$ for all $p \in M$. Then Assumption $A$ holds for all small enough $\bar{\lambda}>0$.

Proof. We start with the first assertion of Assumption A. For this, consider the map

given by

$$
\mathcal{G}: \mathbb{R} \times C_{\text {Dir }}^{2, \alpha}\left(B_{1}\right) \rightarrow C^{\alpha}\left(B_{1}\right)
$$

$$
\mathcal{G}(\bar{\lambda}, \phi):=\Delta \phi+\bar{\lambda} f(p, \phi)
$$

where $p$ is any fixed point on $M$. Since $\mathcal{G}(0,0)=0$ and the partial derivative

$$
D_{\phi} \mathcal{G}(0,0)=\Delta
$$

is invertible with inverse $C^{\alpha}\left(B_{1}\right) \rightarrow C_{\text {Dir }}^{2, \alpha}\left(B_{1}\right)$, it follows from the implicit function theorem that for all $\bar{\lambda}$ in a small interval centered at 0 there is a unique function $\phi_{p, \bar{\lambda}}$ in a small neighborhood of 0 in $C_{\text {Dir }}^{2, \alpha}\left(B_{1}\right)$ that satisfies the equation $\mathcal{G}\left(\bar{\lambda}, \phi_{p, \bar{\lambda}}\right)=0$. Furthermore it depends smoothly on $p \in M$ and $\bar{\lambda} \in \mathbb{R}$, and it is bounded as

$$
\left\|\phi_{p, \bar{\lambda}}\right\|_{C^{2, \alpha}\left(B_{1}\right)} \leqslant C|\bar{\lambda}| .
$$

Take now a small enough positive $\Lambda_{1} \in \mathbb{R}$. In view of the bound (2.2) one can now assume that

$$
\left\|\phi_{p, \bar{\lambda}}\right\|_{L^{\infty}}<1
$$

for all $\bar{\lambda}<\Lambda_{1}$, so the corresponding function $\phi_{p, \bar{\lambda}}$ then satisfies

$$
\begin{aligned}
-\Delta \phi_{p, \bar{\lambda}} & =\bar{\lambda} f\left(p, \phi_{p, \bar{\lambda}}\right) \\
& \geqslant \bar{\lambda}\left(f(p, 0)-\left\|f_{z}(p, \cdot)\right\|_{L^{\infty}((0,1))}\left\|\phi_{p, \bar{\lambda}}\right\|_{L^{\infty}}\right) \\
& \geqslant \bar{\lambda}\left(f(p, 0)-C^{\prime} \bar{\lambda}\right)>0
\end{aligned}
$$

for some constant $C^{\prime}>0$, provided that $\bar{\lambda}$ is smaller than some positive constant $\Lambda_{1}^{\prime}$. Since $\left.\phi_{p, \bar{\lambda}}\right|_{\partial B_{1}}=0$, the maximum principle then ensures that $\phi_{p, \bar{\lambda}}>0$ in $B_{1}$, and Hopf's boundary point lemma shows that $\partial_{\nu} \phi_{p, \bar{\lambda}}<0$.

Let us now pass to the second point of Assumption A. Let us take $\bar{\lambda}<\Lambda_{2}$, where $\Lambda_{2}$ is chosen so that $0<\Lambda_{2} \leqslant \Lambda_{1}^{\prime}$ and also

$$
\Lambda_{2}\left\|f_{z}\right\|_{L^{\infty}(M \times[0,1])}<\lambda_{1}\left(B_{1}\right)
$$


where $\lambda_{1}\left(B_{1}\right)$ is the first Dirichlet eigenvalue of the unit ball. With a positive $\bar{\lambda}<\Lambda_{2}$ we automatically obtain that $\Delta+\bar{\lambda} f_{z}\left(p, \phi_{p}\right)$ is coercive, i.e., that there is a positive constant $c$ such that

$$
\int_{B_{1}}\left(|\nabla u(x)|^{2}-\bar{\lambda} f_{z}\left(p, \phi_{p, \bar{\lambda}}(x)\right) u(x)^{2}\right) d x \geqslant c\|u\|_{H^{1}\left(B_{1}\right)}^{2}
$$

for all $u \in H_{0}^{1}\left(B_{1}\right)$. It is standard that the inverse of $\Delta+\bar{\lambda} f_{z}\left(p, \phi_{p, \bar{\lambda}}\right)$ with Dirichlet boundary condition then exists as a map $C^{\alpha}\left(B_{1}\right) \rightarrow C^{2, \alpha}\left(B_{1}\right)$.

We now pass to the third point of Assumption A. We are going to prove that the coercitivity condition (2.4) implies the third condition. Equivalently, for each integer $j \geqslant 2$, let $b_{p, j}(r)$ satisfy Equation (2.1) with the boundary conditions $b_{p, j}^{\prime}(0)=0$ and $b_{p, j}(1)=0$. We have to show that $b_{p, j}^{\prime}(1) \neq 0$ for each $j \geqslant 2$.

First we claim that $b_{p, j} \leqslant 0$ for all $j \geqslant 2$. To prove it, let us apply the inequality (2.4) to a radial function $u \in H_{0}^{1}\left(B_{1}\right)$, which we simply denote by $u(r)$, to obtain

$$
\int_{0}^{1}\left(u^{\prime}(r)^{2}-\bar{\lambda} f_{z}\left(p, \phi_{p, \bar{\lambda}}(r)\right) u(r)^{2}\right) r^{n-1} d r \geqslant 0
$$

If $b_{p, j} \geqslant 0$ in an interval $\left[r_{1}, r_{2}\right]$ with $b_{p, j}\left(r_{i}\right)=0$, then multiplying (2.1) by $b_{p, j} r^{n-1}$ and integrating by parts yields

$$
\int_{r_{1}}^{r_{2}}\left(\left(b_{p, j}^{\prime}\right)^{2}-\bar{\lambda} f_{z}\left(p, \phi_{p, \bar{\lambda}}\right) b_{p, j}^{2}+\frac{1}{r^{2}} j(j+n-2) b_{p, j}^{2}\right) r^{n-1} d r \leqslant 0,
$$

which, by (2.5), implies that $b_{p, j} \equiv 0$ on $\left[r_{1}, r_{2}\right]$. If $b_{p, j} \geqslant 0$ in an interval $\left[0, r_{2}\right]$ with $b_{p, j}\left(r_{2}\right)=0$, a similar argument (using $b_{p, j}^{\prime}(0)=0$ ) shows that $b_{p, j} \equiv 0$ on $\left[0, r_{2}\right]$. Altogether, this proves the claim.

Suppose now that $b_{p, j}^{\prime}(1)=0$, for some $j \geqslant 2$. Then, since $b_{p, j}(1)=0$ and $b_{p, j} \leqslant 0$ for all $j \geqslant 2$, we have $b_{p, j}^{\prime \prime}(1) \leqslant 0$, but evaluating (2.1) at $r=1$ yields

$$
\begin{aligned}
0 & =(n-1) b_{p, j}^{\prime}(1)=(n-1-j(j+n-2)) \phi_{p, \bar{\lambda}}^{\prime}(1)-b_{p, j}^{\prime \prime}(1) \\
& \geqslant(n-1-j(j+n-2)) \phi_{p, \bar{\lambda}}^{\prime}(1)>0,
\end{aligned}
$$

where we have used that

$$
\phi_{p, \bar{\lambda}}^{\prime}(1)=\left.\partial_{\nu} \phi_{p, \bar{\lambda}}\right|_{\partial B_{1}}<0
$$

by Hopf's boundary point lemma. This gives a contradiction. Therefore, $b_{p, j}^{\prime}(1)>0$ for all $j \geqslant 2$. This completes the proof of the theorem upon noticing that all the smallness conditions that we impose on $\bar{\lambda}$ are uniform in $p \in M$ by the compactness of $M$.

Now, our aim in the rest of this section is to study the second class of nonlinearities considered in Theorem 1.1 namely those functions $f(p, z)$ that vanish at $z=0$ and satisfy certain additional assumptions. In order to show that these nonlinearities satisfy Assumption A (which is done in Theorem 2.5 below), we need to introduce some preliminary definitions and results. These will also play an important role later in Section 3 .

Let us assume that a nonlinearity $f \in C_{\text {loc }}^{1, \alpha}(M \times \mathbb{R})$ satisfies conditions (i) and (ii) in Assumption $\mathrm{A}$, with $\bar{\lambda}=1$. That is, for each point $p \in M$, there exists 
a positive radial function $\phi_{p}(x)$ of class $C^{2, \alpha}$ on the unit Euclidean ball, varying differentiably with $p$ and solving the Dirichlet problem

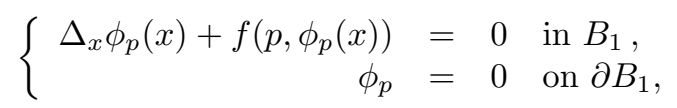

for each $p \in M$. Moreover, for each $p \in M$, the linear operator

$$
L_{p}:=\Delta+f_{z}\left(p, \phi_{p}\right): C_{\text {Dir }}^{2, \alpha}\left(B_{1}\right) \rightarrow C^{0, \alpha}\left(B_{1}\right)
$$

is invertible.

Let $C_{\mathrm{avg}}^{k, \alpha}\left(S^{n-1}\right)$ denote the space of functions on $S^{n-1}$ of class $C^{k, \alpha}$ whose integral on $S^{n-1}$ vanishes. By the invertibility of $L_{p}$ we can consider, for each $w \in C_{\text {avg }}^{2, \alpha}\left(S^{n-1}\right)$, the unique solution $\psi_{w}$ in $C^{2, \alpha}\left(B_{1}\right)$ to

$$
\left\{\begin{aligned}
L_{p} \psi_{w} & =0 & & \text { in } B_{1} \\
\psi_{w} & =-c_{1} w & & \text { on } \partial B_{1}
\end{aligned}\right.
$$

where $c_{1}:=\left.\partial_{r} \phi_{p}\right|_{r=1}$ is the outward radial derivative of $\phi_{p}$ on $\partial B_{1}$. Since $\phi_{p}$ is radial, $c_{1}$ is a constant. The assignment $w \mapsto \psi_{w}$ allows us to define the operator

$$
\begin{aligned}
H_{p}: \quad C_{\text {avg }}^{2, \alpha}\left(S^{n-1}\right) & \rightarrow C_{\text {avg }}^{1, \alpha}\left(S^{n-1}\right) \\
w & \left.\mapsto\left(\partial_{r} \psi_{w}+c_{2} w\right)\right|_{\partial B_{1}},
\end{aligned}
$$

where $c_{2}:=\left.\partial_{r}^{2} \phi_{p}\right|_{r=1}$ is another constant. Indeed, we will see that $H_{p}$ takes values in $C_{\mathrm{avg}}^{1, \alpha}\left(S^{n-1}\right)$.

Denote by $\Delta_{S^{n-1}}$ the Laplace-Beltrami operator of the unit sphere $S^{n-1}$ of $\mathbb{R}^{n}$. It is well known that the eigenvalues of $-\Delta_{S^{n-1}}$ are $\mu_{j}:=j(n+j-2)$ with $j$ ranging over the nonnegative integers. Denote by $V_{j}$ the corresponding eigenspaces, which are given by the restriction to the unit sphere of the space of harmonic polynomials on $\mathbb{R}^{n}$ that are homogeneous of degree $j$. Then we have:

Proposition 2.4. The operator $H_{p}$ satisfies the following properties:

(a) $H_{p}: C_{\text {avg }}^{2, \alpha}\left(S^{n-1}\right) \rightarrow C_{\text {avg }}^{1, \alpha}\left(S^{n-1}\right)$ is a self-adjoint, first order elliptic operator that preserves the eigenspaces of $-\Delta_{S^{n-1}}$.

(b) It can be defined alternatively by

$$
H_{p}(w)=\left.\partial_{r} \Psi_{w}\right|_{\partial B_{1}},
$$

where, for each $w \in C_{\mathrm{avg}}^{2, \alpha}\left(S^{n-1}\right), \Psi_{w}$ is the unique continuous solution to

$$
\left\{\begin{aligned}
L_{p} \Psi_{w} & =\frac{1}{r^{2}} \partial_{r} \phi_{p}\left(\Delta_{S^{n-1}}+n-1\right) w & & \text { in } B_{1} \\
\Psi_{w} & =0 & & \text { on } \partial B_{1} .
\end{aligned}\right.
$$

(c) The kernel of $H_{p}$ contains the eigenspace $V_{1}$ of $-\Delta_{S^{n-1}}$ with eigenvalue $n-1$.

(d) The following three statements are equivalent:

(1) $\operatorname{ker} H_{p}=V_{1}$,

(2) $f$ satisfies condition (iii) of Assumption $A$ with $\bar{\lambda}=1$,

(3) there exists a constant $c_{p}>0$ such that $\left\|H_{p}(w)\right\|_{C^{1, \alpha}\left(S^{n-1}\right)} \geqslant c_{p}\|w\|_{C^{2, \alpha}\left(S^{n-1}\right)}$ for all $w \in C^{2, \alpha}\left(S^{n-1}\right) \cap\left(V_{0} \oplus V_{1}\right)^{\perp}$.

Proof. The operator $H_{p}$ is a linear combination of the Dirichlet-to-Neumann operator for $L_{p}=\Delta+f_{z}\left(p, \phi_{p}\right)$ and the identity. Hence, $H_{p}$ is elliptic of first order, 
essentially self-adjoint on the space of smooth functions, and lower bounded, meaning that $H_{p} \geqslant-C$ in the sense that

$$
\int_{S^{n-1}} w H_{p}(w) d \sigma \geqslant-C\|w\|_{L^{2}}^{2}
$$

for all $w$ in the Sobolev space $H^{1}\left(S^{n-1}\right)$.

In order to prove the other statements, we will first give an alternative description of the operator $H_{p}$. Thus, for each $w \in C_{\text {avg }}^{2, \alpha}\left(S^{n-1}\right)$ we consider the unique continuous solution $\Psi_{w}$ to (2.8); since $\partial_{r} \phi_{p}(0)=0$, standard estimates guarantee the existence of such $\Psi_{w}$ and its regularity away from the origin. Using the formula

$$
\Delta h=\partial_{r}^{2} h+\frac{n-1}{r} \partial_{r} h+\frac{1}{r^{2}} \Delta_{S^{n-1}} h
$$

and the fact that

$$
\Delta \partial_{r} \phi_{p}=-f_{z}\left(p, \phi_{p}\right) \partial_{r} \phi_{p}+\frac{n-1}{r^{2}} \partial_{r} \phi_{p}
$$

some direct calculations show that $\Psi_{w}$ is given by

$$
\Psi_{w}(y)=\psi_{w}(y)+\partial_{r} \phi_{p}(y) w(y /|y|)
$$

for $y \in B_{1}$, where $\psi_{w}$ is the solution to (2.6). Hence, we have that

$$
H_{p}(w)=\left.\partial_{r} \Psi_{w}\right|_{\partial B_{1}}, \quad \text { for each } w \in C_{\text {avg }}^{2, \alpha}\left(S^{n-1}\right) .
$$

This alternative definition easily implies that $H_{p}$ preserves the eigenspaces $V_{j}$ of $-\Delta_{S^{n-1}}$, its image lies in the space of functions with average zero in $S^{n-1}$, and $V_{1} \subset \operatorname{ker} H_{p}$.

In order to conclude the proof we have to show claim (d). Thus, let

$$
w=\sum_{j=1}^{\infty} w_{j},
$$

with $w_{j} \in V_{j}$, be the eigenfunction decomposition of $w \in C_{\mathrm{avg}}^{2, \alpha}\left(S^{n-1}\right)$. Then an easy computation shows that

$$
H_{p}(w)=\sum_{j=1}^{\infty} \alpha_{p, j} w_{j},
$$

where $\alpha_{p, j}=b_{p, j}^{\prime}(1)$ and $b_{p, j}$ is the continuous solution to (2.1) with $b_{p, j}(1)=0$. Since $V_{1} \subset \operatorname{ker} H_{p}$, we have $\alpha_{1}=0$. Condition (iii) of Assumption $\mathrm{A}$ is equivalent to $\alpha_{p, j}=b_{p, j}^{\prime}(1) \neq 0$ for each $j \geqslant 2$, which happens precisely when $V_{1}=\operatorname{ker} H_{p}$. Since $H_{p}$ is elliptic and lower bounded, its eigenvalues satisfy $\lim _{j \rightarrow \infty} \alpha_{p, j}=+\infty$ and, hence, $\operatorname{ker} H_{p}=V_{1}$ if and only if there exists a positive constant $c_{p}$ such that $\left\|H_{p}(w)\right\|_{C^{1, \alpha}\left(S^{n-1}\right)} \geqslant c_{p}\|w\|_{C^{2, \alpha}\left(S^{n-1}\right)}$ for all $w \in C_{\text {avg }}^{2, \alpha}\left(S^{n-1}\right) \cap V_{1}^{\perp}$. This concludes the proof.

Now we are in a position to show that the second kind of nonlinearities mentioned in Theorem 1.1 satisfy Assumption A:

Theorem 2.5. Suppose that $M$ is compact, the function $f$ is of class $C_{\mathrm{loc}}^{2}(M \times \mathbb{R})$ and, for all $p \in M$, it satisfies $f(p, 0)=0, f_{z}(p, 0)=c>0$, where $c$ is independent of $p$, and $f_{z z}(p, 0) \neq 0$. Then Assumption $A$ holds for all $\bar{\lambda}$ in an open interval one of whose endpoints is $\lambda_{1}\left(B_{1}\right) / c$, where $\lambda_{1}\left(B_{1}\right)$ is the first Dirichlet eigenvalue of the Laplacian of the Euclidean unit ball. 
Proof. As in the proof of Theorem 2.3 for each fixed $p$, consider the operator $\mathcal{G}: \mathbb{R} \times C_{\text {Dir }}^{2, \alpha}\left(B_{1}\right) \rightarrow C^{\alpha}\left(B_{1}\right)$ given by

$$
\mathcal{G}(\lambda, \phi):=\Delta \phi+\lambda f(p, \phi) .
$$

By assumption, we have that $\mathcal{G}(\lambda, 0)=0$, for all $\lambda \in \mathbb{R}$. Define

$$
\lambda_{0}:=\lambda_{1}\left(B_{1}\right) / f_{z}(p, 0)=\lambda_{1}\left(B_{1}\right) / c,
$$

Then the partial derivative

$$
D_{\phi} \mathcal{G}\left(\lambda_{0}, 0\right)=\Delta+\lambda_{1}\left(B_{1}\right)
$$

is an operator from $C_{\text {Dir }}^{2, \alpha}\left(B_{1}\right)$ to $C^{\alpha}\left(B_{1}\right)$ whose kernel is generated by the first eigenfunction $\varphi_{1}$ of the Dirichlet Laplacian of $B_{1}$ (which is normalized to have unit $L^{2}$-norm), and whose range is the subspace $\left\langle\varphi_{1}\right\rangle^{\perp}$ of functions in $C^{\alpha}\left(B_{1}\right)$ that are $L^{2}$-orthogonal to $\varphi_{1}$, so in particular it has codimension one in $C^{\alpha}\left(B_{1}\right)$. We have used here the well-known fact that the first eigenvalue has multiplicity one. Moreover, by assumption we have that the second partial derivative

$$
D_{\lambda \phi} \mathcal{G}\left(\lambda_{0}, 0\right) \varphi_{1}=c \varphi_{1} \neq 0
$$

does not belong to the range $\left\langle\varphi_{1}\right\rangle^{\perp}$ of $D_{\phi} \mathcal{G}\left(\lambda_{0}, 0\right)=\Delta+\lambda_{1}\left(B_{1}\right)$. Then we are in the conditions of Crandall-Rabinowitz Theorem [8, Lemma 1.1], whence we obtain the existence of $\lambda_{p, s} \in \mathbb{R}$ and $\phi_{p, s} \in C_{\text {Dir }}^{2, \alpha}\left(B_{1}\right)$, both continuously differentiable depending on $s \in\left(-s_{0}, s_{0}\right)$ and on $p \in M$, such that $\lambda_{p, 0}=\lambda_{0}, \phi_{p, 0}=0$, and $\mathcal{G}\left(\lambda_{p, s}, \phi_{p, s}\right)=0$, that is,

$$
\Delta \phi_{p, s}+\lambda_{p, s} f\left(p, \phi_{p, s}\right)=0 .
$$

Moreover, the same result guarantees that $\phi_{p, s}=s\left(\varphi_{1}+\widetilde{\phi}_{p, s}\right)$ where $\widetilde{\phi}_{p, s} \in\left\langle\varphi_{1}\right\rangle^{\perp}$ is a $C^{1}$-curve with $\widetilde{\phi}_{p, 0}=0$. Thus, for all $s \in\left(0, s_{0}\right)$ (possibly taking a smaller $\left.s_{0}\right)$, the function $\phi_{p, s}$ is positive in $B_{1}$, and also $f\left(p, \phi_{p, s}\right)>0$ in $B_{1}$. Then, by Hopf's boundary point lemma, $\left.\partial_{\nu} \phi_{p, s}\right|_{B_{1}}<0$. Hence, for each fixed $s \in\left(0, s_{0}\right), \phi_{p, s}$ satisfies item (i) in Assumption A for $\bar{\lambda}=\lambda_{p, s}$.

Now, [8, Corollary 1.13] guarantees the existence of $\varphi_{p, \lambda} \in C_{\text {Dir }}^{2, \alpha}\left(B_{1}\right)$ and $\gamma_{p}(\lambda) \in$ $\mathbb{R}$, both depending continuously differentiably on $\lambda$ in an open interval around $\lambda_{0}$, and of $v_{p, s} \in C_{\text {Dir }}^{2, \alpha}\left(B_{1}\right)$ and $\mu_{p, s} \in \mathbb{R}$, both depending continuously differentiably on $s \in\left(-s_{0}, s_{0}\right)$, such that

$$
\left(\Delta+\lambda f_{z}(p, 0)\right) \varphi_{p, \lambda}=\gamma_{p}(\lambda) \varphi_{p, \lambda}
$$

and

$$
L_{p, s} v_{p, s}=\mu_{p, s} v_{p, s}
$$

where $L_{p, s}$ is the linear operator given by

$$
L_{p, s}:=\Delta+\lambda_{p, s} f_{z}\left(p, \phi_{p, s}\right) .
$$

Moreover,

$$
\gamma_{p}\left(\lambda_{0}\right)=0=\mu_{p, 0}, \quad \varphi_{p, \lambda_{0}}=v_{p, 0}=\varphi_{1}, \quad v_{p, s}-\varphi_{1} \in\left\langle\varphi_{1}\right\rangle^{\perp} \cap C_{\text {Dir }}^{2, \alpha}\left(B_{1}\right),
$$

and $\mu_{p, s}$ is a simple eigenvalue of $L_{p, s}$. Now, if the nonlinearity satisfies $f_{z z}(p, 0)<0$ for all $p \in M$, then $\lambda_{p, s}>\lambda_{0}$ for all $s>0$ small enough (see [27, Corollary 1.1]). If $f_{z z}(p, 0)>0$ for all $p \in M$, then $\lambda_{p, s}<\lambda_{0}$ for all $s>0$ small enough (see [27, Remark 1.4]). In any case, the derivative $\partial_{s} \lambda_{p, s}$ cannot be identically zero in any interval around $s=0$. Since [8, Theorem 1.16] guarantees that $\mu_{p, s}$ and $-s \partial_{s} \lambda_{p, s}$ 
have the same zeroes near $s=0$, it follows that $\mu_{p, s} \neq 0$ for infinitely many $s$ near $s=0$. Then, the same result states that

$$
\lim _{\substack{s \rightarrow 0 \\ \mu_{p, s} \neq 0}} \frac{-s \partial_{s} \lambda_{p, s} \gamma^{\prime}\left(\lambda_{0}\right)}{\mu_{p, s}}=1,
$$

which, by L'Hôpital's rule, implies

$$
-\left(\partial_{s} \lambda_{p, 0}\right) \gamma^{\prime}\left(\lambda_{0}\right)=\partial_{s} \mu_{p, 0}
$$

where $\partial_{s} \lambda_{p, 0}$ and $\partial_{s} \mu_{p, 0}$ denote the partial derivatives of $\lambda_{p, s}$ and $\mu_{p, s}$ with respect to $s$ at $s=0$, respectively.

Relation (2.9) can be rewritten as $(\Delta+a(\lambda)) \varphi_{p, \lambda}=0$, where $a(\lambda)=\lambda f_{z}(p, 0)-$ $\gamma_{p}(\lambda) \in \mathbb{R}$. Then, $a(\lambda)$ is an eigenvalue of $-\Delta$ with Dirichlet boundary conditions. Since $a\left(\lambda_{0}\right)=\lambda_{1}\left(B_{1}\right)$, by the discreteness of the eigenvalues of $-\Delta$ and the continuity of $a(\lambda)$ with respect to $\lambda$, we deduce that that $a(\lambda)=\lambda_{1}\left(B_{1}\right)$ for all $\lambda$ close to $\lambda_{0}$, that is

$$
\gamma_{p}(\lambda)=\lambda f_{z}(p, 0)-\lambda_{1}\left(B_{1}\right)
$$

for all $\lambda$ in a neighborhood of $\lambda_{0}$. Hence,

$$
\gamma_{p}^{\prime}\left(\lambda_{0}\right)=f_{z}(p, 0)=c>0 .
$$

Similarly, since $\mu_{p, 0}=0$ is the smallest eigenvalue of $-L_{p, 0}=-\left(\Delta+\lambda_{1}\left(B_{1}\right)\right)$, we have that $\mu_{p, s}$ is also the smallest eigenvalue of $-L_{p, s}$, for all $s \in\left(-s_{0}, s_{0}\right)$. Hence, taking a smaller $s_{0}$ if necessary, for each $s \in\left(-s_{0}, s_{0}\right), L_{p, s}: C_{\text {Dir }}^{2, \alpha}\left(B_{1}\right) \rightarrow C^{0, \alpha}\left(B_{1}\right)$ is invertible if and only if $\mu_{p, s} \neq 0$. Let us show that this indeed holds for all small enough $s>0$.

By differentiating (2.10) with respect to $s$ we get

$$
\begin{aligned}
0= & \Delta \partial_{s} v_{p, s}+\partial_{s} \lambda_{p, s} f_{z}\left(p, \phi_{p, s}\right) v_{p, s}+\lambda_{p, s} f_{z z}\left(p, \phi_{p, s}\right) \partial_{s} \phi_{p, s} v_{p, s} \\
& +\lambda_{p, s} f_{z}\left(p, \phi_{p, s}\right) \partial_{s} v_{p, s}-\partial_{s} \mu_{p, s} v_{p, s}-\mu_{p, s} \partial_{s} v_{p, s}
\end{aligned}
$$

which, evaluated at $s=0$, multiplied by $\varphi_{1}$ and integrating in $B_{1}$, yields

$$
0=f_{z}(p, 0) \partial_{s} \lambda_{p, 0}+\lambda_{0} f_{z z}(p, 0) \int_{B_{1}} \varphi_{1}^{3}-\partial_{s} \mu_{p, 0} .
$$

This, together with (2.11), (2.12) and the assumption $f_{z z}(p, 0) \neq 0$, implies that

$$
\partial_{s} \lambda_{p, 0}=-\frac{1}{2 c} \lambda_{0} f_{z z}(p, 0) \int_{B_{1}} \varphi_{1}^{3} \neq 0
$$

where we are using that $\varphi_{1}>0$. Indeed, $\lambda_{p, s}$ is strictly increasing in $s$ if $f_{z z}(p, 0)<$ 0 , whereas $\lambda_{p, s}$ is strictly decreasing in $s$ if $f_{z z}(p, 0)>0$, for $s \in\left(-s_{0}, s_{0}\right)$, maybe taking a smaller $s_{0}$. Taking into account that $\mu_{p, s}$ and $-s \partial_{s} \lambda_{p, s}$ have the same zeroes, we deduce that $\mu_{p, s} \neq 0$ for all $s \in\left(s_{0}, s_{0}\right) \backslash\{0\}$. Thus, $L_{p, s}: C_{\text {Dir }}^{2, \alpha}\left(B_{1}\right) \rightarrow$ $C^{0, \alpha}\left(B_{1}\right)$ is invertible, which proves item (ii) in Assumption A.

Fix $p \in M$ and $s \in\left(-s_{0}, s_{0}\right) \backslash\{0\}$. Similarly as in the discussion before Proposition 2.4, for each $w \in C_{\mathrm{avg}}^{2, \alpha}\left(S^{n-1}\right)$ consider the unique solution $\psi_{p, s, w} \in C^{2, \alpha}\left(B_{1}\right)$ to

$$
\left\{\begin{array}{rlll}
L_{p, s} \psi_{p, s, w} & =0 & & \text { in } B_{1} \\
\psi_{p, s, w} & =-c_{1} w & & \text { on } \partial B_{1}
\end{array}\right.
$$

where $c_{1}:=\left.\partial_{r} \phi_{p, s}\right|_{r=1} \in \mathbb{R}$. 
Now, for each $p \in M$ and $s \in\left(-s_{0}, s_{0}\right) \backslash\{0\}$, associated to $L_{p, s}$ we consider the operator $H_{p, s}$ defined analogously as in (2.7). By Proposition 2.4, $H_{p, s}$ can be defined alternatively by

$$
H_{p, s}(w)=\left.\partial_{r} \Psi_{p, s, w}\right|_{\partial B_{1}}
$$

where, for each $w \in C_{\text {avg }}^{2, \alpha}\left(S^{n-1}\right)$ and $s \neq 0, \Psi_{p, s, w}$ is the unique continuous solution to

$$
\left\{\begin{aligned}
L_{p, s} \Psi_{p, s, w} & =\frac{1}{r^{2}} \partial_{r} \phi_{p, s}\left(\Delta_{S^{n-1}}+n-1\right) w & & \text { in } B_{1} \\
\Psi_{p, s, w} & =0 & & \text { on } \partial B_{1} .
\end{aligned}\right.
$$

For $s \neq 0$ we can write

$$
\Psi_{p, s, w}=s \eta_{p, s, w},
$$

for some $\eta_{p, s, w} \in C_{\text {Dir }}^{0}\left(B_{1}\right)$. Using this, the identity $\phi_{p, s}=s\left(\varphi_{1}+\widetilde{\phi}_{p, s}\right)$, and dividing by $s$, 2.14) implies that $\eta_{p, s, w}$ is the only continuous solution to

$$
\left\{\begin{aligned}
L_{p, s} \eta_{p, s, w} & =\frac{1}{r^{2}} \partial_{r}\left(\varphi_{1}+\widetilde{\phi}_{p, s}\right)\left(\Delta_{S^{n-1}}+n-1\right) w & & \text { in } B_{1} \\
\eta_{p, s, w} & =0 & & \text { on } \partial B_{1}
\end{aligned}\right.
$$

for each $s \neq 0$ and $w \in C_{\text {avg }}^{2, \alpha}\left(S^{n-1}\right)$.

Since $v_{p, s}$ is a positive function in $B_{1}$, a result by Gidas, Ni and Nirenberg [16. Theorem 1] guarantees that $v_{p, s}$ is radial, and hence, $\left.\partial_{r} v_{p, s}\right|_{r=1}$ is constant on $S^{n-1}$. Then, by the second equation in (2.13) and since $\int_{S^{n-1}} w=0$, we have

$$
\int_{S^{n-1}} \psi_{p, s, w} \partial_{r} v_{p, s}=-\int_{S^{n-1}} c_{1} w \partial_{r} v_{p, s}=0 .
$$

Now, by taking the product of the first equation in 2.13) with $v_{p, s} \in C_{\text {Dir }}^{2, \alpha}\left(B_{1}\right)$, integrating by parts and using (2.10) and (2.16), we have

$$
\begin{aligned}
0 & =\int_{B_{1}} v_{p, s} L_{p, s} \psi_{p, s, w} \\
& =\int_{B_{1}} \psi_{p, s, w} \Delta v_{p, s}-\int_{S^{n-1}} \psi_{p, s, w} \partial_{r} v_{p, s}+\int_{B_{1}} \lambda_{p, s} f_{z}\left(p, \phi_{p, s}\right) \psi_{p, s, w} v_{p, s} \\
& =\int_{B_{1}} \psi_{p, s, w}\left(\mu_{p, s}-\lambda_{p, s} f_{z}\left(p, \phi_{p, s}\right)\right) v_{p, s}+\int_{B_{1}} \lambda_{p, s} f_{z}\left(p, \phi_{p, s}\right) \psi_{p, s, w} v_{p, s} \\
& =\mu_{p, s} \int_{B_{1}} v_{p, s} \psi_{p, s, w} .
\end{aligned}
$$

Thus, if $\mu_{p, s} \neq 0$, then $\psi_{p, s, w}$ is $L^{2}$-orthogonal to $v_{p, s}$. It follows that $\eta_{p, s, w}$ is also $L^{2}$-orthogonal to $v_{p, s}$, and therefore to the kernel of $L_{p, s}$, for each $s \neq 0$.

Since $L_{p, 0}=\Delta+\lambda_{1}\left(B_{1}\right)$ and $\widetilde{\phi}_{p, 0}=0$, one can now take the limit $s \rightarrow 0$ in (2.15) to deduce that

$$
\eta_{p, 0, w}:=\lim _{s \rightarrow 0} \eta_{p, s, w}
$$

is the unique continuous solution in $\left\langle\varphi_{1}\right\rangle^{\perp}$ to

$$
\left\{\begin{aligned}
\Delta \eta_{p, 0, w}+\lambda_{1}\left(B_{1}\right) \eta_{p, 0, w} & =\frac{1}{r^{2}} \partial_{r} \varphi_{1}\left(\Delta_{S^{n-1}}+n-1\right) w & & \text { in } B_{1} \\
\eta_{p, 0, w} & =0 & & \text { on } \partial B_{1} .
\end{aligned}\right.
$$

Now, for each $s \in\left(-s_{0}, s_{0}\right)$ we define

$$
\widetilde{H}_{p, s}(w):=\left.\partial_{r} \eta_{p, s, w}\right|_{\partial B_{1}} .
$$

Hence $H_{p, s}(w)=s \widetilde{H}_{p, s}(w)$, for all $s \in\left(-s_{0}, s_{0}\right)$ and $w \in C_{\text {avg }}^{2, \alpha}\left(S^{n-1}\right)$. 
The boundary problem (2.17) is precisely the one in [31, (4.7)], and the operator $\tilde{H}_{p, 0}$ is the one in [31, (4.8)]. It was proved in 31] that $\left\|\tilde{H}_{p, 0}(w)\right\|_{C^{1, \alpha}\left(S^{n-1}\right)} \geqslant$ $C\|w\|_{C^{2, \alpha}\left(S^{n-1}\right)}$ for all $w$ that is $L^{2}$-orthogonal to $V_{0} \oplus V_{1}$, for some constant $C \in \mathbb{R}$. By the continuity of $H_{p, s}$ with respect to $s$ we have that $\left\|\tilde{H}_{p, s}(w)\right\|_{C^{1, \alpha}\left(S^{n-1}\right)} \geqslant$ $\frac{C}{2}\|w\|_{C^{2, \alpha}\left(S^{n-1}\right)}, s \in\left(-s_{0}, s_{0}\right)$, maybe taking a smaller $s_{0}$. Hence

$$
\left\|H_{p, s}(w)\right\|_{C^{1, \alpha}\left(S^{n-1}\right)}=s\left\|\tilde{H}_{p, s}(w)\right\|_{C^{1, \alpha}\left(S^{n-1}\right)} \geqslant \frac{C s}{2}\|w\|_{C^{2, \alpha}\left(S^{n-1}\right)},
$$

for all $s \in\left(-s_{0}, s_{0}\right)$ and $w \in C_{\text {avg }}^{2, \alpha}\left(S^{n-1}\right) \cap V_{1}^{\perp}$. Therefore, by Proposition 2.4(d) the nonlinearity $f$ satisfies condition (iii) of Assumption A with $\bar{\lambda}=\lambda_{p, s}$ and $\phi_{p}=\phi_{p, s}$, for each $s \in\left(-s_{0}, s_{0}\right) \backslash\{0\}$. This concludes the proof of the theorem upon observing that all the smallness conditions that we impose on $s$ (and hence on $\left|\lambda_{p, s}-\lambda_{0}\right|$ ) are uniform in $p \in M$ by the compactness of $M$.

\section{Proof of Theorem 1.1}

Our objective in this section is to show that any nonlinearity for which Assumption A holds satisfies the conclusions of Theorem 1.1.

Theorem 3.1. Let $M$ be a compact Riemannian manifold, and $f$ any function in $C_{\text {loc }}^{1, \alpha}(M \times \mathbb{R})$ that satisfies Assumption A. Then for every small enough positive $\varepsilon$ there exists a domain $\Omega \subset M$ with volume equal to $\left|B_{\varepsilon}\right|$ and a positive constant $\lambda$, which is of order $\varepsilon^{-2}$, such that the overdetermined problem (1.1) admits a positive solution. The domain $\Omega$ is $C^{2, \alpha}$-small perturbation of a geodesic ball of radius $\varepsilon$.

In particular, this implies Theorem 1.1 by virtue of Theorems 2.3 and 2.5 .

The proof consists of three steps. As these steps are quite involved, we will include several auxiliary propositions. Just as in the rest of the proof, in these propositions we assume without further mention that Assumption A holds with $\bar{\lambda}=1$ (see Remark 2.2). Many of the arguments we will use in this section are modeled on ideas due to Pacard-Sicbaldi [31] and Delay-Sicbaldi [9].

Step 1: Analysis of the rescaled equation with Dirichlet boundary conditions. Using the notation (1.3), the solution domains that we will construct can be described as $\Omega=B_{\varepsilon(1+v)}^{g}(p)$, where $v: T_{p}^{1} M \rightarrow(-1, \infty)$ is a function on the unit sphere of the tangent space at $p$ of class $C^{2, \alpha}\left(T_{p}^{1} M\right), \varepsilon>0$, and $p \in M$. We will decompose $v$ as a sum $v=v_{0}+\bar{v}$ of a constant $v_{0}$ and a function $\bar{v} \in C_{\text {avg }}^{2, \alpha}\left(T_{p}^{1} M\right)$. But, for the sake of convenience, instead of considering a domain that depends on the function $v=v_{0}+\bar{v}$, we will work on a fixed domain, namely on the unit ball $B_{1}$ of $\mathbb{R}^{n}$, endowed with a metric that depends on $\varepsilon$ and $v$.

In order to do that, we introduce some notation. Fixed a point $p \in M$, let $T_{\varepsilon}$ be the homothety of $T_{p} M$ (which is diffeomorphic to $\mathbb{R}^{n}$ ) given by $T_{\varepsilon}(y)=\varepsilon y$, $y \in T_{p} M$. Given $v \in C^{2, \alpha}\left(T_{p}^{1} M\right)$, we consider the open subset

$$
B_{1+v}:=\left\{x \in T_{p} M:|x|<1+v(x /|x|)\right\}
$$

of $T_{p} M$ and the parametrization $\beta: B_{1} \rightarrow B_{1+v}$ given by

$$
\beta(y):=\left(1+v_{0}+\chi(y) \bar{v}\left(\frac{y}{|y|}\right)\right) y,
$$


where $\chi$ is a fixed radial cutoff function that vanishes for $|y| \leqslant 1 / 2$ and is equal to 1 for $|y| \geqslant 3 / 4$. Then, we define $Y: B_{1} \rightarrow B_{\varepsilon(1+v)}^{g}$ as the composition

$$
Y:=\exp _{p}^{g} \circ T_{\varepsilon} \circ \beta \text {. }
$$

Of course, $\beta$ and $Y$ depend on $p, \varepsilon$ and $v$, but we remove this dependence from the notation for the sake of simplicity. Note that $Y\left(B_{1}\right)=\{p\}$ if $\varepsilon=0$, whereas if $\varepsilon>0$ and $\varepsilon\|1+v\|_{L^{\infty}}$ is less than the injectivity radius $R$ of the manifold at $p$, then $Y$ is a diffeomorphism.

Let $x=\left(x^{1}, \ldots, x^{n}\right)$ be normal coordinates in $B_{R}^{g}(p)$ around $p$, and let $g_{i j}$ be the components of the metric $g$ in these coordinates. Then, for each $\varepsilon \in[0, R / 2]$, we consider the metric $\bar{g}$ whose entries in the coordinates $x=\left(x^{1}, \ldots, x^{n}\right)$ are

$$
\bar{g}_{i j}(x):=g_{i j}(\varepsilon x) .
$$

Note that this metric is smooth in the neighborhood $B_{2}$ of $B_{1}$ in $T_{p} M$, where $B_{r}$ is the ball of radius $r$ in $T_{p} M$ with respect to the metric $g_{p}$ at the point $p$. Equivalently, $\bar{g}$ can be defined in a coordinate-independent fashion as

$$
\bar{g}=\varepsilon^{-2}\left(\exp _{p}^{g} \circ T_{\varepsilon}\right)^{*}\left(\left.g\right|_{B_{R}^{g}(p)}\right) .
$$

Hence, $\bar{g}$ is a metric (on an open subset of $T_{p} M$ ) that is homothetic to the pullback metric $\left(\exp _{p}^{g} \circ T_{\varepsilon}\right)^{*} g$. Moreover, in the limit $\varepsilon \rightarrow 0$ the metric $\bar{g}$ tends to the Euclidean metric $g_{0}$, so we will define $\left.\bar{g}\right|_{\varepsilon=0}$ this way.

For each $p, \varepsilon$ and $v$ such that $\varepsilon\|1+v\|_{L^{\infty}}<R$, we define the metric

$$
\widehat{g}:=\beta^{*} \bar{g}=\varepsilon^{-2} Y^{*} g
$$

on the ball $B_{1} \subset T_{p} M$. Given a nonlinearity $f \in C_{\text {loc }}^{1, \alpha}(M \times \mathbb{R})$, we consider the function $\widehat{f} \in C_{\text {loc }}^{1, \alpha}\left(B_{1} \times \mathbb{R}\right)$ such that

$$
\widehat{f}(y, z)=f(Y(y), z)
$$

for $y \in B_{1}$ and $z \in \mathbb{R}$. In other words, $\widehat{f}(y, z)$ is just the expression of the function $f(p, z)$ in the local coordinates $y$.

Here and henceforth, by taking coordinate charts in $M$, we identify $T_{p} M$ with $\mathbb{R}^{n}$, the unit tangent sphere $T_{p}^{1} M$ of $T_{p} M$ with the unit sphere $S^{n-1}$ of $\mathbb{R}^{n}$, and the unit ball of $T_{p} M$ (with respect to the inner product $g_{p}$ ) with $B_{1} \subset \mathbb{R}^{n}$.

Proposition 3.2. There exists $\delta_{0}>0$ such that for each positive $\delta<\delta_{0}, p \in$ $M, \varepsilon \in[0, \delta)$, and $\bar{v} \in C_{\text {avg }}^{2, \alpha}\left(T_{p}^{1} M\right)$ with $\|\bar{v}\|_{C^{2, \alpha}\left(T_{p}^{1} M\right)}<\delta$, there exist a unique constant $v_{0}=v_{0}(p, \varepsilon, \bar{v}) \in(-C \delta, C \delta)$ and a unique positive function $\widehat{u}=\widehat{u}(p, \varepsilon, \bar{v}) \in$ $C^{2, \alpha}\left(B_{1}\right)$ with $\left\|\widehat{u}-\phi_{p}\right\|_{C^{2, \alpha}\left(B_{1}\right)}<C \delta$, and such that

$$
\operatorname{vol}_{\widehat{g}}\left(B_{1}\right)=\left|B_{1}\right|
$$

with $v:=v_{0}+\bar{v}$, and

$$
\left\{\begin{array}{rlll}
\Delta_{\widehat{g}} \widehat{u}+\widehat{f}(\cdot, \widehat{u}) & = & 0 & \text { in } B_{1} \\
\widehat{u} & = & 0 & \text { on } \partial B_{1} .
\end{array}\right.
$$

Here $C$ is a uniform constant. Moreover, $\widehat{u}$ and $v_{0}$ depend smoothly on $p, \varepsilon$ and $\bar{v}$. If $\varepsilon=0$ and $\bar{v}=0$, then $u=\phi_{p}$ and $v_{0}=0$. 
Proof. Observe that if $\varepsilon=0$ and $\bar{v}=0$, then $\widehat{g}=\left(1+v_{0}\right)^{2} g_{0}, d V_{\widehat{g}}=\left(1+v_{0}\right)^{n} d V$, where $g_{0}$ is the Euclidean metric, $d V$ is the Euclidean volume form, and $\widehat{f}(y, z)=$ $f(p, z)$, for $y \in B_{1}, z \in \mathbb{R}$; in particular, we have that $\Delta_{\widehat{g}} \widehat{u}=\left(1+v_{0}\right)^{-2} \Delta \widehat{u}$. Thus, if $\varepsilon=0$ and $\bar{v}=0$, by Assumption A a solution to (3.4) satisfying (3.3) is given by $\widehat{u}=\phi_{p}$ and $v_{0}=0$.

Let us recall that $C_{\text {Dir }}^{2, \alpha}\left(B_{1}\right)$ is the subspace of functions in $C^{2, \alpha}\left(B_{1}\right)$ that vanish on the boundary of $B_{1}$. Let $\mathcal{U}$ be an open neighborhood of $(0,0,0)$ in $[0, \infty) \times$ $C_{\text {avg }}^{2, \alpha}\left(S^{n-1}\right) \times \mathbb{R}$ such that $\varepsilon\left(1+\bar{v}+v_{0}\right)$ is less than the injectivity radius of $(M, g)$, for all $\left(\varepsilon, \bar{v}, v_{0}\right) \in \mathcal{U}$. Then the map

$$
\begin{aligned}
\mathcal{N}: \quad M \times \mathcal{U} \times C_{\mathrm{Dir}}^{2, \alpha}\left(B_{1}\right) & \rightarrow C^{0, \alpha}\left(B_{1}\right) \times \mathbb{R} \\
\left(p, \varepsilon, \bar{v}, v_{0}, \psi\right) & \mapsto\left(\Delta_{\widehat{g}} \psi+\widehat{f}(\cdot, \psi), \operatorname{vol}_{\widehat{g}}\left(B_{1}\right)-\left|B_{1}\right|\right)
\end{aligned}
$$

where $|\cdot|$ denotes the Euclidean volume, is smooth. Since $\widehat{g}=\left(1+v_{0}\right)^{2} g_{0}$ and $\Delta_{\widehat{g}} \psi=\left(1+v_{0}\right)^{-2} \Delta \psi$ for $\varepsilon=0$ and $\bar{v}=0$, we have

$$
\begin{aligned}
\mathcal{N}(p, 0,0,0, \psi) & =(\Delta \psi+f(p, \psi), 0), \\
\mathcal{N}\left(p, 0,0, v_{0}, \phi_{p}\right) & =\left(\left(1+v_{0}\right)^{-2} \Delta \phi_{p}+f\left(p, \phi_{p}\right),\left(\left(1+v_{0}\right)^{n}-1\right)\left|B_{1}\right|\right),
\end{aligned}
$$

for every $p \in M, \psi \in C_{\text {Dir }}^{2, \alpha}\left(B_{1}\right)$ and $v_{0} \in \mathbb{R}$ close enough to zero. Thus, for all $p \in M$, we have

$$
\begin{aligned}
D_{\psi} \mathcal{N}\left(p, 0,0,0, \phi_{p}\right) & =\left(\Delta+f_{z}\left(p, \phi_{p}\right), 0\right), \\
D_{v_{0}} \mathcal{N}\left(p, 0,0,0, \phi_{p}\right) & =\left(2 f\left(p, \phi_{p}\right), n\left|B_{1}\right|\right) .
\end{aligned}
$$

Therefore, since by Assumption A the operator

$$
\Delta+f_{z}\left(p, \phi_{p}\right): C_{\text {Dir }}^{2, \alpha}\left(B_{1}\right) \rightarrow C^{0, \alpha}\left(B_{1}\right)
$$

is invertible, the partial differential

$$
D_{\left(v_{0}, \psi\right)} \mathcal{N}\left(p, 0,0,0, \phi_{p}\right): \mathbb{R} \times C_{\text {Dir }}^{2, \alpha}\left(B_{1}\right) \rightarrow C^{0, \alpha}\left(B_{1}\right) \times \mathbb{R}
$$

is also invertible, for every $p \in M$. Thus, for any fixed $p \in M$, the implicit function theorem guarantees that, for each $(q, \varepsilon, \bar{v})$ in a neighborhood of $(p, 0,0)$ in $M \times[0, \infty) \times C_{\text {avg }}^{2, \alpha}\left(S^{n-1}\right)$, there exists a unique $\left(v_{0}, \widehat{u}\right)$ in a neighborhood of $\left(0, \phi_{p}\right)$ in $\mathbb{R} \times C_{\text {Dir }}^{2, \alpha}\left(B_{1}\right)$ such that $\mathcal{N}\left(q, \varepsilon, \bar{v}, v_{0}, \widehat{u}\right)=(0,0)$. Moreover, since $\phi_{p}>0$ and $\left.\partial_{\nu} \phi_{p}\right|_{\partial B_{1}}<0$, maybe taking a smaller neighborhood we have that $\widehat{u}>0$. Hence, if $\delta$ is a small enough positive constant, which, by the compactness of $M$, means that $\delta$ be smaller than some positive constant $\delta_{0}$ independent of the point $p \in M$, then for each positive constant $\varepsilon<\delta$ and each $\bar{v}$ with $C^{2, \alpha}$-norm smaller than $\delta$, there exist unique $v_{0}$ in a neighborhood of 0 and $\widehat{u} \in C_{\text {Dir }}^{2, \alpha}\left(B_{1}\right)$ in a neighborhood of $\phi_{p}$ satisfying (3.3) and (3.4). Furthermore,

$$
\left|v_{0}\right|+\left\|\widehat{u}-\phi_{p}\right\|_{C^{2, \alpha}\left(B_{1}\right)}<C \delta
$$

with some uniform constant $C$. This completes the proof.

Step 2: Analyzing when the normal derivative is constant. Let $\omega_{n}:=$ $\left|S^{n-1}\right|$ be the area of the $(n-1)$-dimensional unit sphere of $\mathbb{R}^{n}$. We consider the operator

$$
\mathcal{F}(p, \varepsilon, \bar{v}):=\left.\widehat{g}\left(\nabla_{\widehat{g}} \widehat{u}, \nu_{\widehat{g}}\right)\right|_{\partial B_{1}}-\frac{1}{\omega_{n}} \int_{\partial B_{1}} \widehat{g}\left(\nabla_{\widehat{g}} \widehat{u}, \nu_{\widehat{g}}\right) d \sigma
$$


where $\nu_{\widehat{g}}$ is the outward unit normal vector field to $\partial B_{1}$ with respect to the metric $\widehat{g}$, $\left(v_{0}, \widehat{u}\right)$ is the solution provided by Proposition 3.2 and $d \sigma$ is the standard area measure on the unit sphere. As follows from the last part of the proof of Proposition 3.2 $\mathcal{F}$ is well-defined from a neighborhood of $M \times\{0\} \times\{0\}$ in $M \times[0, \infty) \times C_{m}^{2, \alpha}\left(S^{n-1}\right)$ into $C_{\text {avg }}^{1, \alpha}\left(S^{n-1}\right)$. Of course, $\mathcal{F}(p, \varepsilon, \bar{v})=0$ exactly when the normal derivative $\left.\widehat{g}\left(\nabla_{\widehat{g}} \widehat{u}, \nu_{\widehat{g}}\right)\right|_{\partial B_{1}}$ is constant.

Recall from the discussion after the proof of Theorem 2.3 in Section 2 that for each $w \in C_{\text {avg }}^{2, \alpha}\left(S^{n-1}\right)$ we considered the unique (by Assumption A) solution $\psi_{w}$ to

$$
\left\{\begin{aligned}
\Delta \psi_{w}+f_{z}\left(p, \phi_{p}\right) \psi_{w} & =0 & & \text { in } B_{1} \\
\psi_{w} & =-c_{1} w & & \text { on } \partial B_{1},
\end{aligned}\right.
$$

where $c_{1}:=\left.\partial_{r} \phi_{p}\right|_{r=1}$ is a constant. The assignment $w \mapsto \psi_{w}$ allowed us to define the operator

$$
\begin{aligned}
H_{p}: \quad C_{\mathrm{avg}}^{2, \alpha}\left(S^{n-1}\right) & \rightarrow C_{\text {avg }}^{1, \alpha}\left(S^{n-1}\right) \\
w & \left.\mapsto\left(\partial_{r} \psi_{w}+c_{2} w\right)\right|_{\partial B_{1}},
\end{aligned}
$$

where $c_{2}:=\left.\partial_{r}^{2} \phi_{p}\right|_{r=1}$ is another constant.

The reason for which we are interested in the operator $H_{p}$ is that it is the linearization of the operator $\mathcal{F}$ defined in (3.5), as we show below:

Proposition 3.3. The differential of $\mathcal{F}$ with respect to $\bar{v}$ at $\varepsilon=0$ and $\bar{v}=0$ is $H_{p}$, i.e. $D_{\bar{v}} \mathcal{F}(p, 0,0)=H_{p}$, for all $p \in M$.

Proof. Fix $p \in M$. We have to show that $\left.\partial_{s}\right|_{s=0} \mathcal{F}(p, 0, s w)=H_{p}(w)$, for every $w \in C_{\mathrm{avg}}^{2, \alpha}\left(S^{n-1}\right)$. For the moment, let us assume that $f(p, \cdot)$ is a real analytic function of one variable.

We set $\bar{v}=s w$. For $\varepsilon=0$ we have that $\bar{g}=g_{0}$ and $\widehat{g}=\beta^{*} g_{0}$. Then the solution $\widehat{u}$ given in Proposition 3.2 satisfies

$$
\left\{\begin{array}{rlll}
\Delta_{\widehat{g}} \widehat{u}+f(p, \widehat{u}) & =0 & & \text { in } B_{1} \\
\widehat{u} & = & 0 & \text { on } \partial B_{1}
\end{array}\right.
$$

and $\operatorname{vol}_{\widehat{g}}\left(B_{1}\right)=\left|B_{1}\right|$. Since we are assuming that $f(p, \cdot)$ is analytic, the CauchyKowalevski theorem guarantees that the solution $\phi_{p}$ can be extended to an open set containing the closed unit ball of $\mathbb{R}^{n}$, and it satisfies $\Delta \phi_{p}+f\left(p, \phi_{p}\right)=0$ therein. Hence, $\widehat{\phi}_{p}=\phi_{p} \circ \beta$ is a solution to $\Delta_{\widehat{g}} \widehat{\phi}_{p}+f\left(p, \widehat{\phi}_{p}\right)=0$ in $B_{1}$.

By setting $\widehat{\psi}=\widehat{u}-\widehat{\phi}_{p}$ we observe that

$$
\left\{\begin{aligned}
\Delta_{\widehat{g}} \widehat{\psi}-f\left(p, \widehat{\phi}_{p}\right)+f\left(p, \widehat{\psi}+\widehat{\phi}_{p}\right) & =0 & & \text { in } B_{1} \\
\widehat{\psi} & =-\widehat{\phi}_{p} & & \text { on } \partial B_{1} .
\end{aligned}\right.
$$

By construction, $\widehat{\psi}$ and $v_{0}$ depend smoothly on $s$. Moreover, if $s=0$, then $\widehat{g}=g_{0}$, $v_{0}=0, \widehat{\phi}_{p}=\phi_{p}$ and $\widehat{\psi}=0$. Let $\dot{\psi}=\left.\partial_{s} \widehat{\psi}\right|_{s=0}$ and $\dot{v}_{0}=\left.\partial_{s} v_{0}\right|_{s=0}$. Taking derivatives in (3.7) with respect to $s$ and evaluating at $s=0$, we get

$$
\left\{\begin{aligned}
\Delta \dot{\psi}+f_{z}\left(p, \phi_{p}\right) \dot{\psi} & =0 & & \text { in } B_{1} \\
\dot{\psi} & =-c_{1}\left(\dot{v}_{0}+w\right) & & \text { on } \partial B_{1}
\end{aligned}\right.
$$

where $c_{1}=\left.\partial_{r} \phi_{p}\right|_{r=1}$. Now, by differentiating the relation $\operatorname{vol}_{\widehat{g}}\left(B_{1}\right)=\left|B_{1}\right|$ with respect to $s$ at $s=0$, we get

(3.9) $0=\left.\partial_{s}\right|_{s=0} \operatorname{vol}_{\widehat{g}}\left(B_{1}\right)=\left.\partial_{s}\right|_{s=0}\left|B_{1+v}\right|=\int_{S^{n-1}} g_{0}\left(\Xi, \partial_{r}\right) d \sigma=\int_{S^{n-1}}\left(\dot{v}_{0}+w\right) d \sigma$, 
where we have used the differentiation formula for moving regions 14, Appendix C.4], and where $\Xi=\left(\dot{v}_{0}+w\right) \partial_{r}$ is the velocity field of the moving boundary $\partial B_{1+v}$ at $s=0$. Since $\int_{S^{n-1}} w d \sigma=0$, we deduce that $\dot{v}_{0}=0$.

Altogether, we have shown that $v_{0}=\mathcal{O}\left(s^{2}\right)$ and $\widehat{u}=\widehat{\phi}_{p}+s \psi_{w}+\mathcal{O}\left(s^{2}\right)$, where $\psi_{w}$ is the solution to (3.6). Moreover, recalling from (3.1) the definition of $\beta$, in $B_{1} \backslash B_{3 / 4}$ we can write

$$
\begin{aligned}
\widehat{u}(y) & =\phi_{p}\left(((1+s w(y /|y|)) y)+s \psi_{w}(y)+\mathcal{O}\left(s^{2}\right)\right. \\
& =\phi_{p}(y)+s\left(w(y /|y|) r \partial_{r} \phi_{p}+\psi_{w}\right)+\mathcal{O}\left(s^{2}\right),
\end{aligned}
$$

where $r=|y|$. Now, some calculations show that, in polar coordinates $y=r z$, $r>0, z \in S^{n-1}$, the metric $\widehat{g}=\beta^{*} g_{0}$ in $B_{1} \backslash B_{3 / 4}$ adopts the expression

$\widehat{g}=\left(1+v_{0}+s w\right)^{2} d r^{2}+2 s\left(1+v_{0}+s w\right) r d w d r+\left.r^{2}\left(1+v_{0}+s w\right)^{2} g_{0}\right|_{S^{n-1}}+s^{2} r^{2} d w^{2}$.

Using this and the fact that $v_{0}=\mathcal{O}\left(s^{2}\right)$, one gets that the outward unit normal vector field to $\partial B_{1}$ with respect to the metric $\widehat{g}$ is given by

$$
\nu_{\widehat{g}}=\left(1-s w+\mathcal{O}\left(s^{2}\right)\right) \partial_{r}+\mathcal{O}(s) \partial_{z_{j}},
$$

where $\partial_{z_{j}}$ are coordinate vector fields on $S^{n-1}$. This, together with (3.10), yields

$$
\widehat{g}\left(\nabla_{\widehat{g}} \widehat{u}, \nu_{\widehat{g}}\right)=\nu_{\widehat{g}}(\widehat{u})=c_{1}+s\left(c_{2} w+\partial_{r} \psi_{w}\right)+\mathcal{O}\left(s^{2}\right) \quad \text { on } \partial B_{1},
$$

where $c_{2}=\left.\partial_{r}^{2} \phi_{p}\right|_{r=1}$. This concludes the proof in the case that $f(p, \cdot)$ is analytic.

If $f \in C_{\text {loc }}^{1, \alpha}(M \times \mathbb{R})$, let $f_{\delta} \in C^{\omega}(\mathbb{R}), \delta \in\left(0, \delta_{0}\right)$, be a family of analytic functions converging to $f_{0}:=f(p, \cdot)$ locally in the $C^{1, \alpha}$-norm. Consider the smooth map

$$
\begin{aligned}
\mathcal{N}_{p}: \quad\left[0, \delta_{0}\right) \times C_{\text {Dir }}^{2, \alpha}\left(B_{1}\right) & \rightarrow C^{0, \alpha}\left(B_{1}\right) \\
(\delta, \psi) & \mapsto \Delta \psi+f_{\delta} \circ \psi .
\end{aligned}
$$

Then $\mathcal{N}_{p}\left(0, \phi_{p}\right)=\Delta \phi_{p}+f\left(p, \phi_{p}\right)=0$, and moreover

$$
D_{\psi} \mathcal{N}_{p}\left(0, \phi_{p}\right)=\Delta+f_{0}^{\prime} \circ \phi_{p}=\Delta+f_{z}\left(p, \phi_{p}\right)
$$

is invertible as an operator from $C_{\text {Dir }}^{2, \alpha}\left(B_{1}\right)$ to $C^{0, \alpha}\left(B_{1}\right)$ by Assumption A. Hence, the implicit function theorem guarantees the existence of $\phi_{p}^{\delta} \in C_{\text {Dir }}^{2, \alpha}\left(B_{1}\right)$ smoothly depending on $\delta \in\left[0, \delta_{0}\right)$, for a maybe smaller $\delta_{0}>0$, such that $\Delta \phi_{p}^{\delta}+f_{\delta} \circ \phi_{p}^{\delta}=0$ in $B_{1}$, and $\Delta+f_{\delta}^{\prime} \circ \phi_{p}^{\delta}$ is invertible. Therefore, the arguments above apply to each one of the analytic nonlinearities $f_{\delta}, \delta \in\left(0, \delta_{0}\right)$, with associated solutions $\phi_{p}^{\delta} \in C_{\mathrm{Dir}}^{2, \alpha}\left(B_{1}\right)$ to $\Delta \phi_{p}^{\delta}+f_{\delta} \circ \phi_{p}^{\delta}=0$, and associated operators $\mathcal{F}^{\delta}$ and $H_{p}^{\delta}$. Thus, $D_{\bar{v}} \mathcal{F}^{\delta}(p, 0,0)=H_{p}^{\delta}$ for each $\delta \in\left(0, \delta_{0}\right)$. Since both $\mathcal{F}^{\delta}$ and $H_{p}^{\delta}$ depend continuously on $\delta$, we deduce that

$$
D_{\bar{v}} \mathcal{F}(p, 0,0)=\lim _{\delta \rightarrow 0} D_{\bar{v}} \mathcal{F}^{\delta}(p, 0,0)=\lim _{\delta \rightarrow 0} H_{p}^{\delta}=H_{p},
$$

which concludes the proof.

Let us now recall that the first eigenspace of $-\Delta_{S^{n-1}}, V_{1}$, is the restriction to the unit sphere of the space of linear functions on $T_{p} M$, which one can write as

$$
\langle a, \cdot\rangle
$$

with $a \in T_{p} M$. Using this fact together with Propositions 3.3 and 2.4 combined with some ideas about the center of mass, one can prove the following result via the implicit function theorem: 
Proposition 3.4. There exists $\varepsilon_{0}>0$ such that, for each $\varepsilon \in\left[0, \varepsilon_{0}\right)$ and each $p \in M$, there is a unique $\bar{v}_{\varepsilon, p} \in C_{\mathrm{avg}}^{2, \alpha}\left(T_{p}^{1} M\right)$ and a unique $a_{\varepsilon, p} \in T_{p} M$, both smoothly depending on $p$ and $\varepsilon$, such that $\left\|\bar{v}_{\varepsilon, p}\right\|_{C^{2, \alpha}\left(T_{p}^{1} M\right)}<\varepsilon_{0}$,

$$
\mathcal{F}\left(p, \varepsilon, \bar{v}_{\varepsilon, p}\right)+\left\langle a_{\varepsilon, p}, \cdot\right\rangle=0 \quad \text { on } \partial B_{1}=S^{n-1},
$$

and the center of mass of $\partial B_{\varepsilon\left(1+v_{0}+\bar{v}\right)}^{g}(p)$ is $p$. Moreover, $\bar{v}_{0, p}=0, a_{0, p}=0$, and the map

$$
p \in M \mapsto a_{\varepsilon, p} \in T_{p} M
$$

defines a $C^{1, \alpha}$-vector field $a_{\varepsilon}$ on $M$.

Proof. In [29, Lemma 2.7] it was proved that there exists a smooth map $A$ such that $\left(\exp _{p}^{g}\right)^{-1}(c(p, \varepsilon, v))=\varepsilon A(p, \varepsilon, v)$, where $c(p, \varepsilon, v)$ denotes the center of mass of $\partial B_{\varepsilon(1+v)}^{g}(p)$ as defined in the Introduction, and

$$
A(p, 0, v)=\frac{\int_{S^{n-1}}(1+v(z))^{n-1} z \sqrt{|\nabla v(z)|^{2}+(1+v(z))^{2}} d z}{\int_{S^{n-1}}(1+v(z))^{n-2} \sqrt{|\nabla v(z)|^{2}+(1+v(z))^{2}} d z} .
$$

Define the operator $Q=\mathrm{Id}-\Pi_{1}$, where $\Pi_{1}$ is the orthogonal projection of $L^{2}\left(S^{n-1}\right)$ onto the first eigenspace $V_{1}$ of the spherical Laplacian $\Delta_{S^{n-1}}$. We consider the smooth map

$$
\overline{\mathcal{F}}(p, \varepsilon, \bar{v})=\left(A\left(p, \varepsilon, \bar{v}+v_{0}(p, \varepsilon, \bar{v})\right), Q \mathcal{F}(p, \varepsilon, \bar{v})\right)
$$

defined from a neighborhood of $M \times\{(0,0)\}$ in $M \times[0, \infty) \times C_{\text {avg }}^{2, \alpha}\left(S^{n-1}\right)$ into a neighborhood of $(0,0)$ in $\mathbb{R}^{n} \times\left(C_{\text {avg }}^{1, \alpha}\left(S^{n-1}\right) \cap V_{1}^{\perp}\right)$. Using (3.11), by considering the Taylor expansions up to order one of the numerator and denominator in $A(p, 0, t \bar{v}+$ $\left.v_{0}(p, 0, t \bar{v})\right)\left(\right.$ cf. [29, Lemma 2.9]) and taking into account that $v_{0}(p, 0, t \bar{v})=\mathcal{O}\left(t^{2}\right)$ (as follows from (3.9)), one easily shows that

$$
\left.\frac{d}{d t}\right|_{t=0} A\left(p, 0, t \bar{v}+v_{0}(p, 0, t \bar{v})\right)=\frac{n}{\omega_{n}} \int_{S^{n-1}} \bar{v}(z) z d z .
$$

Hence, the differential of the second component of $\overline{\mathcal{F}}$ with respect to $\bar{v}$ at the point $(p, 0,0)$ has image $\mathbb{R}^{n}$ and kernel contained in $V_{1}^{\perp}$. Moreover, we have that $\overline{\mathcal{F}}(p, 0,0)=(0,0)$ and, according to Proposition 3.3. the differential of the second component of $\overline{\mathcal{F}}$ with respect to $\bar{v}$ at $(p, 0,0)$ is $Q H_{p}$, whose kernel is $V_{1}$, and whose image is $C_{\text {avg }}^{1, \alpha}\left(S^{n-1}\right) \cap V_{1}^{\perp}$, by Proposition 2.4. Altogether we deduce that the differential $D_{\bar{v}} \overline{\mathcal{F}}(p, 0,0)$ is invertible. Hence, the implicit function theorem and the compactness of $M$ guarantee the existence, for each $p \in M$ and for all $\varepsilon$ smaller than certain $\varepsilon_{0}$, of a unique $\bar{v}_{\varepsilon, p}$, smoothly depending on $p$ and $\varepsilon$, with $\left\|\bar{v}_{\varepsilon, p}\right\|_{C_{\text {avg }}^{2, \alpha}\left(T_{p}^{1} M\right)}<\varepsilon_{0}$, such that $\overline{\mathcal{F}}\left(p, \varepsilon, \bar{v}_{\varepsilon, p}\right)=(0,0)$. Taking the unique $a_{\varepsilon, p} \in T_{p} M$ such that $\Pi_{1}\left(\mathcal{F}\left(p, \varepsilon, \bar{v}_{\varepsilon, p}\right)\right)+\left\langle a_{\varepsilon, p}, \cdot\right\rangle=0$, where $\langle\cdot, \cdot\rangle$ is the inner product on $T_{p} M$ determined by $g$, the result follows.

Step 3: Existence of zeros of the vector field $a_{\varepsilon}$ and conclusion of the proof. In the previous section we have constructed, for each $\varepsilon \in\left(0, \varepsilon_{0}\right)$ and for each $p \in M$, a function $v_{\varepsilon, p}=v_{0}\left(\varepsilon, p, \bar{v}_{\varepsilon, p}\right)+\bar{v}_{\varepsilon, p} \in C^{2, \alpha}\left(T_{p} M\right)$, a vector $a_{\varepsilon, p} \in T_{p} M$ and a function $\widehat{u}_{\varepsilon, p} \in C_{\text {Dir }}^{2, \alpha}\left(B_{1}\right)$ such that the center of mass of $\partial B_{\varepsilon\left(1+v_{\varepsilon, p}\right)}^{g}(p)$ is $p$, 
$\operatorname{vol}_{\widehat{g}}\left(B_{1}\right)=\left|B_{1}\right|$, and

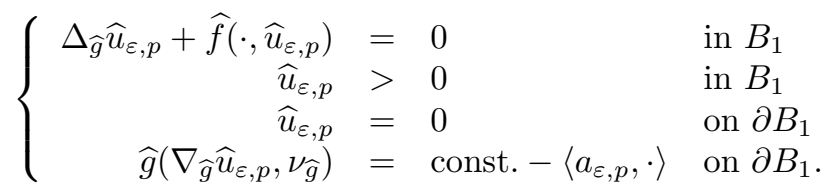

For each $\varepsilon>0$ we define the homothetic metric $g_{\varepsilon}=\varepsilon^{-2} g$ on $M$. Then, for each $p \in M$ and $\varepsilon \in\left(0, \varepsilon_{0}\right)$, we consider the $C^{2, \alpha}$-domain

$$
\Omega_{\varepsilon, p}:=B_{\varepsilon\left(1+v_{\varepsilon, p}\right)}^{g}(p)=B_{1+v_{\varepsilon, p}}^{g_{\varepsilon}}(p)
$$

of $M$, which is centered at $p$. Using the parametrization $Y: B_{1} \rightarrow \Omega_{\varepsilon, p}$ introduced in (3.2), we can define the function $u_{\varepsilon, p} \in C_{\text {Dir }}^{2, \alpha}\left(\Omega_{\varepsilon, p}\right)$ by means of the relation $\widehat{u}_{\varepsilon, p}=: u_{\varepsilon, p} \circ Y$. Recalling that $\widehat{g}=\varepsilon^{-2} Y^{*} g=Y^{*} g_{\varepsilon}$ and $\widehat{f}(y, z)=f(Y(y), z)$, we get that

$$
\Delta_{\widehat{g}} \widehat{u}_{\varepsilon, p}+\widehat{f}\left(\cdot, \widehat{u}_{\varepsilon, p}\right)=\Delta_{Y^{*} g_{\varepsilon}}\left(u_{\varepsilon, p} \circ Y\right)+f\left(Y(\cdot), u_{\varepsilon, p} \circ Y\right)=\Delta_{g_{\varepsilon}} u_{\varepsilon, p}+f\left(\cdot, u_{\varepsilon, p}\right),
$$

and

$$
\operatorname{vol}_{g}\left(B_{\varepsilon\left(1+v_{\varepsilon, p}\right)}^{g}(p)\right)=\varepsilon^{n} \operatorname{vol}_{\bar{g}}\left(B_{1+v_{\varepsilon, p}}\right)=\varepsilon^{n} \operatorname{vol}_{\widehat{g}}\left(B_{1}\right)=\varepsilon^{n}\left|B_{1}\right|=\left|B_{\varepsilon}\right| .
$$

Hence, we can reformulate what we have obtained as follows. For each $\varepsilon \in\left(0, \varepsilon_{0}\right)$ and $p \in M$, we have a collection of $C^{2, \alpha}$-domains $\left\{\Omega_{\varepsilon, p}\right\}_{p \in M}$ of $M$, where $\Omega_{\varepsilon, p}=$ $B_{1+v_{\varepsilon, p}}^{g_{\varepsilon}}(p)$ is centered at $p$ and $\operatorname{vol}_{g}\left(\Omega_{\varepsilon, p}\right)=\left|B_{\varepsilon}\right|$, as well as a collection of solutions $\left\{u_{\varepsilon, p}\right\}_{p \in M}$ to the problem

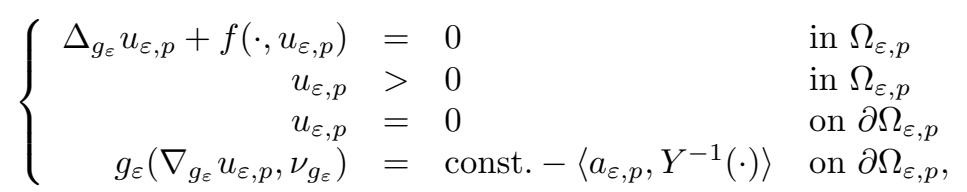

where $\nu_{g_{\varepsilon}}=Y_{*} \nu_{\widehat{g}}$ is the outward $g_{\varepsilon}$-unit normal vector field to $\partial \Omega_{\varepsilon, p}$.

Now we are interested in finding some point $p \in M$ such that the vector $a_{\varepsilon, p} \in T_{p} M$, provided by Proposition 3.4 and appearing in the Neumann condition in (3.14), is zero. Note that, as $\Delta_{g_{\varepsilon}}=\varepsilon^{2} \Delta_{g}$, this would be equivalent to saying that $u_{\varepsilon, p}$ is a solution to the overdetermined problem (1.1) in the domain $\Omega_{\varepsilon, p}$ with $\lambda:=\varepsilon^{-2}$.

To show the existence of zeros of the vector field $a_{\varepsilon}$ we will use a variational technique. Given a function $u \in H_{0}^{1}(\Omega)$, we define its energy as

$$
J(u):=\int_{\Omega}\left(\frac{1}{2}\left|\nabla_{g} u\right|_{g}^{2}-F(\cdot, u)\right) d V_{g},
$$

where

$$
F(p, z):=\int_{0}^{z} f(p, \zeta) d \zeta .
$$

Let $\Omega_{0}$ be a smooth bounded domain of $M$. We call $\left\{\Omega_{t}\right\}_{t \in\left(0, t_{0}\right)}$ a deformation of $\Omega_{0}$ if there exists a smooth vector field $\Xi$ such that $\Omega_{t}=\xi\left(t, \Omega_{0}\right)$, where $\xi(t, \cdot)$ is the flow of $\Xi$. 
Proposition 3.5. Assume that $\left\{\Omega_{t}:=\xi\left(t, \Omega_{0}\right)\right\}_{t \in\left(-t_{0}, t_{0}\right)}$ is a deformation of a smooth bounded domain $\Omega_{0}$, and $u_{t} \in C^{2}\left(\Omega_{t}\right)$ is a solution to the Dirichlet problem $\Delta_{g} u_{t}+f\left(\cdot, u_{t}\right)=0$ in $\Omega_{t}, u_{t}=0$ on $\partial \Omega_{t}$, depending smoothly on $t$. Then the derivative of the energy functional $J(t):=J\left(u_{t}\right)$ at $t=0$ reads as

$$
J^{\prime}(0)=-\frac{1}{2} \int_{\partial \Omega_{0}} g\left(\nabla_{g} u_{0}, \nu_{0}\right)^{2} g\left(\Xi, \nu_{0}\right) d V_{g}
$$

where $\nu_{0}$ is the outward unit normal vector field on $\partial \Omega_{0}$.

Proof. Using the differentiation formula for moving regions (see [14, Appendix C.4]), we get

$$
\begin{aligned}
J^{\prime}(0)= & \int_{\Omega_{0}} g\left(\nabla_{g} \partial_{t} u_{0}, \nabla_{g} u_{0}\right) d V_{g}-\int_{\Omega_{0}} f\left(\cdot, u_{0}\right) \partial_{t} u_{0} d V_{g} \\
& +\int_{\partial \Omega_{0}}\left(\frac{1}{2} g\left(\nabla_{g} u_{0}, \nu_{0}\right)^{2}-F\left(\cdot, u_{0}\right)\right) g\left(\Xi, \nu_{0}\right) d \sigma_{g}
\end{aligned}
$$

where $\partial_{t} u_{0}$ stands for the derivative of $u_{t}$ with respect to $t$ at $t=0, \Xi$ is the vector field defining the deformation, and $d \sigma_{g}$ is the induced Riemannian area measure on $\partial \Omega_{0}$. Integrating by parts in the first integral and using the relation $\Delta_{g} u_{0}+f\left(\cdot, u_{0}\right)=0$, we have

$$
\begin{aligned}
\int_{\Omega_{0}} g\left(\nabla_{g} \partial_{t} u_{0}, \nabla_{g} u_{0}\right) d V_{g} & =-\int_{\Omega_{0}} \partial_{t} u_{0} \Delta_{g} u_{0} d V_{g}+\int_{\partial \Omega_{0}} \partial_{t} u_{0} g\left(\nabla_{g} u_{0}, \nu_{0}\right) d \sigma_{g} \\
& =\int_{\Omega_{0}} f\left(\cdot, u_{0}\right) \partial_{t} u_{0} d V_{g}+\int_{\partial \Omega_{0}} \partial_{t} u_{0} g\left(\nabla_{g} u_{0}, \nu_{0}\right) d \sigma_{g} .
\end{aligned}
$$

Denoting by $\xi$ the flow generated by $\Xi$, by definition we have that $u_{t}(\xi(t, p))=0$ for all $p \in \partial \Omega_{t}$. Differentiating this identity with respect to $t$ at $t=0$ we obtain $\partial_{t} u_{0}=-g\left(\nabla_{g} u_{0}, \Xi\right)$ on $\partial \Omega_{0}$. But, since $u_{0}$ is constant on $\partial \Omega_{0}$, we can write $\partial_{t} u_{0}=-g\left(\nabla_{g} u_{0}, \nu_{0}\right) g\left(\Xi, \nu_{0}\right)$ on $\partial \Omega_{0}$. Moreover, $F\left(\cdot, u_{0}\right)=F(\cdot, 0)=0$ on $\partial \Omega_{0}$. Altogether, we obtain the formula in the statement.

Now, using the solutions $u_{\varepsilon, p}$ to the Dirichlet problem on the domain $\Omega_{\varepsilon, p}$ to (3.14), for each $\varepsilon>0$ small enough we can define a smooth function $\mathcal{J}_{\varepsilon}: M \rightarrow \mathbb{R}$ as

$$
\mathcal{J}_{\varepsilon}(p):=J\left(u_{\varepsilon, p}\right)=\int_{\Omega_{\varepsilon, p}}\left(\frac{1}{2}\left|\nabla_{g_{\varepsilon}} u_{\varepsilon, p}\right|_{g_{\varepsilon}}^{2}-F\left(\cdot, u_{\varepsilon, p}\right)\right) d V_{g_{\varepsilon}}, \quad p \in M .
$$

The following proposition provides a key result to derive the main theorems of this paper:

Proposition 3.6. For each small enough $\varepsilon>0, u_{\varepsilon, p}$ is a solution to the overdetermined problem (1.1) on the domain $\Omega_{\varepsilon, p}$ with $\lambda:=\varepsilon^{-2}$ if and only if $p$ is a critical point of $\mathcal{J}_{\varepsilon}$.

Proof. In view of (3.14), $u_{\varepsilon, p}$ is a solution to the overdetermined problem (1.1) on the domain $\Omega_{\varepsilon, p}$ with $\lambda:=\varepsilon^{-2}$ if and only if $a_{\varepsilon, p}=0$. We start by calculating the differential of $\mathcal{J}_{\varepsilon}$. Let $w \in T_{p} M$ and define $q_{t}=\exp _{p}^{g_{\varepsilon}}(t w)$. If $t$ is small enough, $\partial \Omega_{\varepsilon, q_{t}}$ can be expressed as a graph of a function $h_{t}$ over $\partial \Omega_{\varepsilon, p}$. As the domains have fixed volume, this yields a volume-preserving vector field $\Xi$ on $\partial \Omega_{\varepsilon, p}$ given by

$$
\Xi=\left.\frac{\partial h_{t}}{\partial t}\right|_{t=0} \nu_{g_{\varepsilon}}
$$


Then, by Proposition 3.5 and (3.13) we have

$$
\begin{aligned}
D_{p} \mathcal{J}_{\varepsilon}(w) & =\left.\frac{d}{d t}\right|_{t=0} \mathcal{J}_{\varepsilon}\left(q_{t}\right)=-\frac{1}{2} \int_{\partial \Omega_{\varepsilon, p}} g_{\varepsilon}\left(\nabla_{g_{\varepsilon}} u_{\varepsilon, p}, \nu_{g_{\varepsilon}}\right)^{2} g_{\varepsilon}\left(\Xi, \nu_{g_{\varepsilon}}\right) d \sigma_{g_{\varepsilon}} \\
& =-\frac{1}{2} \int_{\partial B_{1}}\left(b_{\varepsilon, p}-\left\langle a_{\varepsilon, p}, \cdot\right\rangle\right)^{2} \widehat{g}(\widehat{\Xi}, \nu \widehat{g}) d \sigma_{\widehat{g}}
\end{aligned}
$$

where $b_{\varepsilon, p}$ is a constant, $\widehat{\Xi}=Y^{*} \Xi$ and $\nu_{\widehat{g}}=Y^{*} \nu_{g_{\varepsilon}}$. Observe that if $a_{\varepsilon, p}=0$, then $p$ is a critical point of $\mathcal{J}_{\varepsilon}$, since $\widehat{\Xi}$ is volume-preserving.

In order to prove the converse, assume that $D_{p} \mathcal{J}_{\varepsilon}=0$. Then (3.15) implies

$$
2 b_{\varepsilon, p} \int_{\partial B_{1}}\left\langle a_{\varepsilon, p}, \cdot\right\rangle \widehat{g}\left(\widehat{\Xi}, \nu_{\widehat{g}}\right) d \sigma_{\widehat{g}}=\int_{\partial B_{1}}\left\langle a_{\varepsilon, p}, \cdot\right\rangle^{2} \widehat{g}(\widehat{\Xi}, \nu \widehat{g}) d \sigma_{\widehat{g}}
$$

for all $w \in T_{p} M$. By the Taylor expansion of the metric, for all $\varepsilon$ small enough there exists a constant $c$, independent of $\varepsilon$, such that

$$
|\widehat{g}(\widehat{\Xi}, \nu \widehat{g})-\langle w, \cdot\rangle| \leqslant c \varepsilon|w| .
$$

If we choose $w:=b_{\varepsilon, p} a_{\varepsilon, p}$ we have that

$$
\widehat{g}\left(\widehat{\Xi}, \nu_{\widehat{g}}\right)=b_{\varepsilon, p}\left\langle a_{\varepsilon, p}, \cdot\right\rangle+\varepsilon k,
$$

where

$$
|k| \leqslant c\left|b_{\varepsilon, p}\right|\left|a_{\varepsilon, p}\right|
$$

Using this in (3.16) we deduce that there exists a constant $C>0$ (independent of $\varepsilon$ ) such that for all $\varepsilon$ small enough the following inequality holds:

$$
2 b_{\varepsilon, p}^{2} \int_{\partial B_{1}}\left\langle a_{\varepsilon, p}, \cdot\right\rangle^{2} d V_{\widehat{g}} \leqslant C\left|b_{\varepsilon, p}\right|\left(\varepsilon\left|a_{\varepsilon, p}\right|^{3}+\left|a_{\varepsilon, p}\right|^{3}+\varepsilon\left|a_{\varepsilon, p}\right|^{2}\right) .
$$

Since for $\varepsilon$ small enough the left hand side is bounded from below by a positive constant times $b_{\varepsilon, p}^{2}\left|a_{\varepsilon, p}\right|^{2}$, we obtain

$$
b_{\varepsilon, p}^{2}\left|a_{\varepsilon, p}\right|^{2} \leqslant C\left|b_{\varepsilon, p}\right|\left(\varepsilon\left|a_{\varepsilon, p}\right|+\left|a_{\varepsilon, p}\right|+\varepsilon\right)\left|a_{\varepsilon, p}\right|^{2},
$$

for some other constant $C>0$. Now, as $b_{\varepsilon, p} \neq 0$ when $\varepsilon=0$ (because, by Assumption A(i), $\left.\left.\partial_{\nu} \phi_{p}\right|_{\partial B_{1}}<0\right)$, we have that $\left|b_{\varepsilon, p}\right|>C$ for some uniform constant $C>0$. Since $\lim _{\varepsilon \rightarrow 0} a_{\varepsilon, p}=0$, the inequality (3.17) implies that, for $\varepsilon$ small enough, $a_{\varepsilon, p}=0$. Finally observe that the existence of an $\varepsilon_{0}>0$ independent of $p \in M$ is guaranteed by the compactness of $M$ and the fact that the constants in the arguments above are bounds on quantities that only depend on the norms of the geometric objects involved.

This completes the proof of Theorem 3.1 ,

\section{VARIations AND APPlications of the PRoOF of TheOREM 1.1}

In this section we derive some corollaries of the proof of Theorem 3.1. In particular, we prove Theorem 1.2 and a uniqueness result that will be important in the following sections. 
Proof of Theorem 1.2. Let us now replace the equation $\Delta_{g} u+\lambda f(\cdot, u)=0$ by the more general equation

$$
\Delta_{g} u+\left\langle\nabla_{g} u, X\right\rangle+\lambda f(\cdot, u)=0,
$$

where $X$ is a $C^{\alpha}$-vector field on the manifold. Our goal now is to show that if Assumption A holds (notice that this assumption does not involve the vector field $X$ ) and the Euler characteristic of $M$ is nonzero, then the overdetermined boundary problem for Equation (4.1) admits a nontrivial solution on domains that are small perturbations of small geodesic balls.

Going through the proof of Theorem 3.1 one easily checks that the analysis of the rescaled equation developed in Step 1 carries over to the more general equation (4.1), as does the discussion of the behavior of the normal derivative in Step 2. However, the proof of the fact that the vector field $a_{\varepsilon}$ has zeros, presented in Step 3, does not apply to this more general equation, as the demonstration relies on the variational formulation of the equation $\Delta_{g} u+\lambda f(\cdot, u)=0$. However, since we have already shown that $a_{\varepsilon}$ is a $C^{1, \alpha}$-vector field on $M$ (Proposition [3.4), $a_{\varepsilon}$ is guaranteed to have zeros if $M$ is a compact manifold of nonzero Euler characteristic. Theorem 1.2 then follows.

The construction method we have developed implies the following uniqueness result:

Proposition 4.1. Suppose that the nonlinearity $f$ satisfies Assumption A. Suppose that we have two solutions to the overdetermined problem (1.1), given by $(u, \Omega:=$ $\left.B_{\varepsilon(1+v)}^{g}(p), \lambda:=\varepsilon^{-2}\right)$ and $\left(u^{\prime}, \Omega^{\prime}:=B_{\varepsilon\left(1+v^{\prime}\right)}^{g}(p), \lambda:=\varepsilon^{-2}\right)$, respectively. Then, if

(i) $\operatorname{vol}_{g}(\Omega)=\operatorname{vol}_{g}\left(\Omega^{\prime}\right)$,

(ii) $\Omega$ and $\Omega^{\prime}$ are centered at $p$,

(iii) $\varepsilon$ and the $C^{2, \alpha}$-norms of $v, v^{\prime}, \widehat{u}-\phi_{p}$ and $\widehat{u}^{\prime}-\phi_{p}$ are small enough (i.e., smaller than some quantity $\delta$ depending on $p$ ),

then $u=u^{\prime}$ and $\Omega=\Omega^{\prime}$. Moreover, if $M$ is compact, then $\delta>0$ can be taken to be independent of the point $p \in M$.

Proof. In the proof of Proposition 3.2 we could have introduced a new variable $\eta \in(-1,1)$ in the definition of the map $\mathcal{N}$, and substituted the second component of $\mathcal{N}$ by $\operatorname{vol}_{\widehat{g}}\left(B_{1}\right)-\left|B_{1+\eta}\right|$. Then, the implicit function theorem would apply in the same way at the point $\left(p, 0,0,0,0, \phi_{p}\right)$ to derive the existence of unique $v_{0}=$ $v_{0}(p, \varepsilon, \eta, \bar{v}) \in \mathbb{R}$ and $\widehat{u}=\widehat{u}(p, \varepsilon, \eta, \bar{v}) \in C_{\text {Dir }}^{2, \alpha}\left(B_{1}\right)$ in small enough neighborhoods of $0 \in \mathbb{R}$ and of $\phi_{p} \in C_{\text {Dir }}^{2, \alpha}\left(B_{1}\right)$, respectively, satisfying $\mathcal{N}\left(p, \varepsilon, \eta, \bar{v}, v_{0}, \widehat{u}\right)=(0,0)$, whenever $\varepsilon, \eta$ and $\bar{v}$ have small enough norm. Then, the remaining arguments in Section 3 are valid only with some formal modifications (e.g. the operator $\mathcal{F}$ should now be defined as a function of $(p, \varepsilon, \eta, \bar{v})$, and Proposition 3.4 would yield $a_{\varepsilon, \eta, p}$ and $\left.\bar{v}_{\varepsilon, \eta, p}\right)$. In particular, the arguments in Proposition 3.4 imply the uniqueness of $a_{\varepsilon, \eta, p}$ and $\bar{v}_{\varepsilon, \eta, p}$ in small enough neighborhoods of 0 in $T_{p} M$ and $C_{\text {avg }}^{2, \alpha}\left(S^{n-1}\right)$, respectively, whenever $\varepsilon$ and $\eta$ are small enough, say less than $\delta$. Hence, the statement of the corollary holds if, in addition to the hypotheses, we have $\operatorname{vol}_{g}\left(B_{\varepsilon(1+v)}^{g}(p)\right)=$ $\operatorname{vol}_{g}\left(B_{\varepsilon\left(1+v^{\prime}\right)}^{g}(p)\right)=\left|B_{\varepsilon(1+\eta)}\right|$, for some $\eta \in(-\delta, \delta)$. But if $\varepsilon,\|\bar{v}\|_{C^{2, \alpha}\left(S^{n-1}\right)}$ and $\left\|\bar{v}^{\prime}\right\|_{C^{2, \alpha}\left(S^{n-1}\right)}$ are small enough, there exists $\eta \in(-\delta, \delta)$ satisfying the additional condition on the volumes. 


\section{OVERDETERMINED PROBLEMS ON NONCOMPACT MANIFOLDS}

The purpose of this section is to discuss the possibility of relaxing the compactness assumption on $M$ in the results above. Thus, in the rest of this section, $M$ is not assumed to be compact.

A first result is that Theorem 1.1 holds also if the compactness assumption on $M$ is substituted by a suitable homogeneity assumption. To present a rigorous formulation of this fact, consider a group $\mathbf{G}$ of isometries of $(M, g)$ that leave $f$ invariant, namely

$$
\mathbf{G} \subseteq\{\varphi \in \operatorname{Isom}(M): f(\varphi(p), z)=f(p, z), \text { for all } p \in M, z \in \mathbb{R}\} .
$$

Consider the orbit space $M / \mathbf{G}$, that is, the set of all the orbits

$$
\mathbf{G} \cdot p=\{\varphi(p): \varphi \in \mathbf{G}\},
$$

with $p \in M$, of the isometric $\mathbf{G}$-action on $M$, endowed with the quotient topology induced by the canonical projection map $M \rightarrow M / \mathbf{G}, p \mapsto \mathbf{G} \cdot p$ (see [3, §2.1] for more information on isometric actions). Notice that $M / \mathbf{G}$ does not have to be a differentiable manifold: it can be a manifold with corners, or even an orbifold.

The fact that Theorem 1.1 remains valid if the orbit space $M / \mathbf{G}$ is compact (but not necessarily $M$ ) follows from the following proposition. Of course, particularly simple yet important examples of problems to which this result applies are noncompact homogeneous spaces (or, more generally, spaces with a co-compact isometry group) with a position-independent nonlinearity $G(z)$. Recall that a Riemannian manifold $M$ is called homogeneous if its isometry group acts transitively on $M$ or, equivalently, if given any two points $p, q \in M$ there exists an isometry of $M$ mapping $p$ to $q$.

Proposition 5.1. Theorem 3.1 and Proposition 4.1 remain true if the compactness assumption on $M$ is substituted by the following two hypotheses:

(a) the orbit space $M / \mathbf{G}$ is compact,

(b) the map $\phi_{p}$ in Assumption $A$ is $\mathbf{G}$-invariant (i.e., $\phi_{p}=\phi_{\varphi(p)}$ for all $\varphi \in \mathbf{G}$ ).

Moreover, Theorems 2.3] and 2.5, and hence Theorem 1.1, remain true if the compactness assumption on $M$ is substituted by condition (a).

Proof. By definition, the maps $\mathcal{N}$ and $\mathcal{F}$ considered in Section 3 are invariant under the isometries in G. Thus, the compactness arguments used in Section 3 (only in the proofs of Propositions 3.2 and 3.4 can be carried out under the assumption that $M / \mathbf{G}$ is compact and $\phi_{p}$ is $\mathbf{G}$-invariant. Also, by construction, the objects involved in the proof of Proposition 3.6 (namely $\mathcal{J}_{\varepsilon}, a_{\varepsilon, p}, b_{\varepsilon_{p}}$ ) are $\mathbf{G}$-invariant, and hence the proof carries over to the case that $M / \mathbf{G}$ is compact. Similarly, the proofs of Theorems 2.3 and 2.5 remain valid under the hypothesis (a) since $\mathcal{G}$ is $\mathbf{G}$-invariant, giving rise to a $\mathbf{G}$-invariant function $\phi_{p}$ satisfying Assumption A.

Moreover, compactness was needed to ensure the existence of critical points of the functions $\mathcal{J}_{\varepsilon}$. But, since $\mathcal{J}_{\varepsilon}$ is invariant under $\mathbf{G}$, it induces a continuous map $\widetilde{\mathcal{J}}_{\varepsilon}: M / \mathbf{G} \rightarrow \mathbb{R}$. Any preimage under the projection map $M \rightarrow M / \mathbf{G}$ of a maximum point for $\widetilde{\mathcal{J}}_{\varepsilon}$ is also a maximum point for $\mathcal{J}_{\varepsilon}$, and hence, a critical point for $\mathcal{J}_{\varepsilon}$. This shows that Theorem 3.1 holds under the assumptions (a) and (b). Combining 
this with the generalization of Theorems 2.3 and 2.5. we deduce that Theorem 1.1 holds under condition (a).

Remark 5.2. With the same arguments as above one can show that Theorems 1.1 2.3, 2.5 and 3.1, and Proposition 4.1, hold, not only for homogeneous spaces, but also for locally uniformly homogeneous spaces, that is, for those Riemannian manifolds $(M, g)$ such that there exist $R>0$ satisfying that, for any $p, q \in M$, there exists an isometry $\varphi: B_{R}^{g}(p) \rightarrow B_{R}^{g}(q)$. In this case, one has to restrict to positionindependent nonlinearities $f(p, z)=G(z)$.

Next we shall show how to extend the previous results to a broader family of noncompact manifolds that do not need to have any global isometries and whose nonlinearities can depend on the point in the manifold, but for which, roughly speaking, there is a kind of homogeneous behavior at infinity. The geometry at infinity of the manifold $M$ will be modeled by some homogeneous Riemannian manifold $M_{\infty}$ with metric $g_{\infty}$. In order to formalize this idea, let us introduce the appropriate terminology.

Definition 5.3. We will say that the Riemannian $n$-manifold $(M, g)$ is asymptotically homogeneous if there exists an $n$-dimensional homogeneous Riemannian manifold $M_{\infty}$ with metric $g_{\infty}$, a point $p_{\infty} \in M_{\infty}, R>0$, and a function $\varphi$ : $M \times B_{R}^{g_{\infty}}\left(p_{\infty}\right) \rightarrow M$ with the property that for each $\delta>0$ there exists a relatively compact domain $M_{\delta}$ of $M$ such that for all $p \in M \backslash \bar{M}_{\delta}, \varphi_{p}:=\varphi(p, \cdot)$ defines a $C^{2, \alpha}$-diffeomorphism $\varphi_{p}: B_{R}^{g_{\infty}}\left(p_{\infty}\right) \rightarrow B_{R}^{g}(p)$ and $\left\|\varphi_{p}^{*} g-g_{\infty}\right\|_{C^{2, \alpha}\left(B_{R}^{g \infty}\left(p_{\infty}\right)\right)}<\delta$.

Observe that, with this definition, the injectivity radius of an asymptotically homogeneous manifold is always larger than some positive constant $R$.

Remark 5.4. The notion we have just introduced can be regarded as a broad generalization of other well-known concepts. For example, if the model manifold $\left(M_{\infty}, g_{\infty}\right)$ in Definition 5.3 is a Euclidean space, then the above definition means that $(M, g)$ is asymptotically flat. Notice that, although there are several definitions of asymptotically flat manifolds in the literature, they usually imply the notion of asymptotically homogeneous manifold, as is the case, for instance, of Schoen and Yau's definition [35. Similarly, the notion of asymptotically hyperbolic manifold (see e.g. [22]) corresponds to taking as model manifold $\left(M_{\infty}, g_{\infty}\right)$ the real hyperbolic space. In any case, the choice of the point $p_{\infty}$ is inessential due to the isometries of $\left(M_{\infty}, g_{\infty}\right)$.

Now we need to define an analogous notion of "asymptotic homogeneity" for the nonlinearity. To this end we will make the following assumption:

Assumption B. $M$ is an asymptotically homogeneous manifold as above, the nonlinearity $f \in C_{\text {loc }}^{1, \alpha}(M \times \mathbb{R})$ satisfies Assumption A with radial functions $\phi_{p} \in$ $C_{\text {Dir }}^{2, \alpha}\left(B_{1}\right)$ and constant $\bar{\lambda}$, and additionally there exist functions $G_{\infty} \in C_{\text {loc }}^{1, \alpha}(\mathbb{R})$ and $\phi_{\infty} \in C_{\text {Dir }}^{2, \alpha}\left(B_{1}\right)$ such that:

(i) For each $\delta>0$ there exists a relatively compact domain $M_{\delta}$ of $M$ such that

$$
\left\|f(p, \cdot)-G_{\infty}\right\|_{C^{1, \alpha}((-N, N))}+\left\|\phi_{p}-\phi_{\infty}\right\|_{C^{2, \alpha}\left(B_{1}\right)}<\delta
$$


for all $p \in M \backslash \bar{M}_{\delta}$, where $N>0$ is such that, for all $p \in M$,

$$
\left\|\phi_{p}\right\|_{L^{\infty}}+\left\|\phi_{\infty}\right\|_{L^{\infty}}<\frac{N}{2} \text {. }
$$

(ii) The function $G_{\infty}$ also satisfies Assumption A with the same constant $\bar{\lambda}$, the corresponding radial function on the unit ball being precisely $\phi_{\infty}$.

The main result in this section is that Theorem 3.1 remains valid for asymptotically homogeneous manifolds with asymptotically homogeneous nonlinearities. It is clear that, just as before, the key is to prove the result under Assumption B. This is what we will do in the proof of the following theorem.

Theorem 5.5. Suppose that Assumption B holds. Then for every small enough positive $\varepsilon$ there exists a domain $\Omega \subset M$ with volume equal to $\left|B_{\varepsilon}\right|$ and a positive constant $\lambda$, which is of order $\varepsilon^{-2}$, such that the overdetermined problem (1.1) admits a nontrivial nonnegative solution. The domain $\Omega$ is a $C^{2, \alpha}$-small perturbation of a geodesic ball of radius $\varepsilon$.

Before proving this result, we state and prove the following consequence of Theorem 5.5. Roughly speaking, it guarantees that Theorem 5.5 can be applied to the appropriate modifications of the two types of nonlinearities studied in Section 2 .

Theorem 5.6. Let $(M, g)$ be an asymptotically homogeneous manifold. Let $f$ be a function in $C_{\mathrm{loc}}^{1, \alpha}(M \times \mathbb{R})$ such that, as $p \rightarrow \infty, f(p, z)$ tends to a certain function $G_{\infty}(z)$ locally in the $C^{1, \alpha}$-norm in the sense that for any $\delta>0$ and any compact interval $I \subset \mathbb{R}$ there exists a relatively compact domain $M_{I, \delta}$ of $M$ such that

$$
\left\|f(p, \cdot)-G_{\infty}\right\|_{C^{1, \alpha}(I)}<\delta,
$$

for all $p \in M \backslash \bar{M}_{I, \delta}$. Suppose that one of the following assumptions on $f$ holds

(i) $\inf _{p \in M} f(p, 0)>0$, or

(ii) $f \in C_{\text {loc }}^{2}(M \times \mathbb{R})$ converges to $G_{\infty} \in C_{\text {loc }}^{2}(\mathbb{R})$ locally in the $C^{2}$-norm, $f(p, 0)=$ $0, f_{z}(p, 0)=c>0$ and $\inf _{p \in M}\left|f_{z z}(p, \cdot)\right|>0$ for all $p \in M$, where $c$ is a constant independent of $p$.

Then for every small enough $\varepsilon$ there exists a domain $\Omega \subset M$ with volume equal to $\left|B_{\varepsilon}\right|$ and a positive constant $\lambda$, which is of order $\varepsilon^{-2}$, such that the overdetermined problem (1.1) admits a nontrivial nonnegative solution. The domain $\Omega$ is a $C^{2, \alpha}$ small perturbation of a geodesic ball of radius $\varepsilon$.

Proof. We just have to prove that the nonlinearity $f$ satisfies Assumption B for each one of the cases (i) and (ii) in the statement.

Since $f$ tends to $G_{\infty}$ locally in the $C^{1, \alpha}$-norm (or in the $C^{2}$-norm for case (ii)), it follows that $G_{\infty}$ satisfies one of the two conditions analyzed in Section 2. Thus, Theorems 2.3 and 2.5 applied to the nonlinearity $G_{\infty}$ (which is independent of $p \in M)$ guarantee the existence of $\phi_{\infty, \bar{\lambda}} \in C_{\text {Dir }}^{2, \alpha}\left(B_{1}\right)$ satisfying Assumption A, with nonlinear eigenvalue $\bar{\lambda}>0$ in an open interval with endpoint 0 for case (i) or $\lambda_{0}=\lambda_{1}\left(B_{1}\right) / c$ for case (ii).

In the proofs of Theorems 2.3 and 2.5. the only point where we needed the compactness assumption was at the very end of the proofs, in order to guarantee that the smallness conditions imposed on $\bar{\lambda}$ (for case (i)) or $\left|\bar{\lambda}-\lambda_{0}\right|$ (for case (ii)) 
were uniform in $p \in M$. Now $M$ is no longer compact, but the function $f$ tends to a function $G_{\infty}$ that satisfies Assumption A. Thus, using the continuous dependence in the implicit function theorem and the Crandall-Rabinowitz theorem used in Theorems 2.3 and 2.5. respectively, both results also hold in the current setting. That is, the nonlinearity $f$ satisfies Assumption A for each $\bar{\lambda}>0$ in an open interval (with endpoint 0 for case (i) or $\lambda_{0}$ for case (ii)) and $\phi_{p, \bar{\lambda}} \in C_{\text {Dir }}^{2, \alpha}\left(B_{1}\right)$ smoothly depending on $p \in M$. Moreover, by construction, the functions $\phi_{\infty, \bar{\lambda}}$ and $\phi_{p, \bar{\lambda}}$ have small $C^{2, \alpha}$-norm. Hence, we can take $N>0$ such that $\left\|\phi_{p, \bar{\lambda}}\right\|_{L^{\infty}}+\left\|\phi_{\infty, \bar{\lambda}}\right\|_{L^{\infty}}<$ $N / 2$, for all $p \in M$ and all $\bar{\lambda}$ in some open interval.

The fact that $\left\|f(p, \cdot)-G_{\infty}\right\|_{C^{1, \alpha}((-N, N))}<\delta$ for all $p$ outside a compact subset $\bar{M}_{\delta}$ of $M$ follows directly from the hypothesis of locally uniform $C^{1, \alpha}$-convergence of $f(p, \cdot)$ to $G_{\infty}$. In view of this convergence, the fact that $\left\|\phi_{p, \bar{\lambda}}-\phi_{\infty, \bar{\lambda}}\right\|_{C^{2, \alpha}\left(B_{1}\right)}$ is also small follows again from the continuous dependence in the arguments used in Theorems 2.3 and 2.5. respectively. This shows that $f$ satisfies Assumption B for each $\bar{\lambda}$ in some open interval.

Notice that Theorem 1.3 in the Introduction is the particular case of the previous Theorem 5.6 for $(M, g)=\left(\mathbb{R}^{n}, g_{0}\right)$, where $g_{0}$ is the Euclidean metric. Clearly, the Euclidean space is a homogeneous Riemannian manifold so, in particular, it is asymptotically homogeneous. Indeed, in this case, in the definition of asymptotic homogeneity one can take $\left(M_{\infty}, g_{\infty}\right)$ to be the Euclidean space $\left(\mathbb{R}^{n}, g_{0}\right)$ and $B_{R}^{g_{\infty}}\left(p_{\infty}\right)$ to be the unit ball centered at the origin. The function $\varphi: \mathbb{R}^{n} \times B \rightarrow \mathbb{R}^{n}$ is

$$
\varphi(p, z):=p+z,
$$

so $\varphi_{p}=\varphi(p, \cdot)$ is an isometry onto its image, for each $p \in \mathbb{R}^{n}$. Similarly, Theorem 5.6 applies to all noncompact homogeneous Riemannian manifolds, including, as a very specific but important case, the hyperbolic spaces. Other important examples of manifolds to which Theorem [5.6 applies are the symmetric spaces of noncompact type, as well as all known examples of noncompact harmonic spaces (see Section [6 for more information on these spaces).

Now we proceed with the proof of Theorem 5.5 where we include a couple of propositions.

Proof of Theorem 5.5. We will follow the ideas of the proof of Theorem 3.1. We start by adapting the proof of Proposition 3.2 to the new setting. Notice that the difficulty is to show that it is possible to take a bound $\delta$ in Proposition 3.2 that does not go to zero as $p \in M$ goes to infinity.

Proposition 5.7. The claims in Proposition 3.2 hold also in the case that the Riemannian manifold $M$ is asymptotically homogeneous.

Proof. Let $\mathcal{M}$ denote the convex open cone of Riemannian metrics inside the Ba-

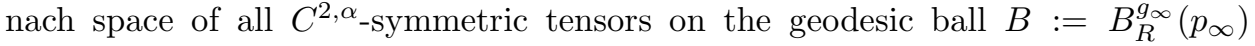
given in Definition 5.3. We will employ the usual notations; for instance, given $h \in \mathcal{M}$ we define $\bar{h}=\varepsilon^{-2}\left(\exp _{p_{\infty}}^{h} \circ T_{\varepsilon}\right)^{*} h$, we consider the parametrization $Y: B_{1} \subset$ $T_{p_{\infty}} B \rightarrow B_{\varepsilon(1+v)}^{h}\left(p_{\infty}\right)$ as in (3.2), the metric $\widehat{h}=\beta^{*} \bar{h}$ on $B_{1}$, and the function 
$\widehat{s}(y, z)=s(Y(y), z), y \in B_{1}, z \in \mathbb{R}$. Consider the smooth map

$$
\begin{aligned}
\mathcal{N}: \mathcal{M}^{\prime} \times C^{1, \alpha}(B \times(-N, N)) \times \mathcal{U} & \rightarrow C^{0, \alpha}\left(B_{1}\right) \times \mathbb{R} \\
\left(h, s, \varepsilon, \bar{v}, v_{0}, \psi\right) & \mapsto\left(\Delta_{\widehat{h}} \psi+\widehat{s}(\cdot, \psi), \operatorname{vol}_{\widehat{h}}\left(B_{1}\right)-\left|B_{1}\right|\right),
\end{aligned}
$$

where $\mathcal{M}^{\prime}$ is an open neighborhood of $g_{\infty}$ in $\mathcal{M}$ and $\mathcal{U}$ is an open neighborhood of $(0,0,0,0)$ in $[0, \infty) \times C_{\text {avg }}^{2, \alpha}\left(S^{n-1}\right) \times \mathbb{R} \times C_{\text {Dir }}^{2, \alpha}\left(B_{1}\right)$ such that $\varepsilon\left(1+\bar{v}+v_{0}\right)<R$ and $\|\psi\|_{L^{\infty}}<N$, for all $h \in \mathcal{M}^{\prime}$ and $\left(\varepsilon, \bar{v}, v_{0}, \psi\right) \in \mathcal{U}$; recall that $N>0$ was introduced in Assumption B.

Just as in the proof of Proposition 3.2, we can apply the implicit function theorem to $\mathcal{N}$ at the point $\left(g_{\infty}, G_{\infty}, 0,0,0, \phi_{\infty}\right)$, where $g_{\infty}$ is the metric of $M_{\infty}, G_{\infty}$ and $\phi_{\infty}$ are as in Assumption B, and $G_{\infty}$ is to be understood as a function defined on $B \times \mathbb{R}$, restricted to $B \times(-N, N)$, but which is independent of the point of $B$. Therefore, for each $(h, s, \varepsilon, \bar{v})$ in a neighborhood $\mathcal{V}$ of $\left(g_{\infty}, G_{\infty}, 0,0\right)$ in $\mathcal{M} \times C^{1, \alpha}(B \times(-N, N)) \times$ $[0, \infty) \times C_{\text {avg }}^{2, \alpha}\left(S^{n-1}\right)$, there exists a unique $\left(v_{0}, \widehat{u}\right)$, smoothly depending on $(h, s, \varepsilon, \bar{v})$, in a neighborhood of $\left(0, \phi_{\infty}\right)$ in $\mathbb{R} \times C_{\text {Dir }}^{2, \alpha}\left(B_{1}\right)$ such that $\mathcal{N}\left(h, s, \varepsilon, \bar{v}, v_{0}, \widehat{u}\right)=0$. We can assume that for some $\delta>0$ the neighborhood $\mathcal{V}$ is given by those $(h, s, \varepsilon, \bar{v})$ satisfying

$$
\max \left\{\left\|h-g_{\infty}\right\|_{C^{2, \alpha}(B)},\left\|s-G_{\infty}\right\|_{C^{1, \alpha}(B \times(-N, N))}, \varepsilon,\|\bar{v}\|_{C^{2, \alpha}\left(S^{n-1}\right)}\right\}<\delta .
$$

For this $\delta$, let $M_{\delta}$ be a relatively compact domain in $M$ that satisfies simultaneously the condition stated in Definition 5.3 and item (i) in Assumption B, and let $M_{\delta}^{\prime} \supset M_{\delta}$ be another relatively compact domain such that if $p \in M \backslash \bar{M}_{\delta}^{\prime}$, then $B_{R}^{g}(p) \subset M \backslash \bar{M}_{\delta}$. Then the smooth maps $\rho: M \backslash \bar{M}_{\delta}^{\prime} \rightarrow \mathcal{M}$ and $\sigma: M \backslash \bar{M}_{\delta}^{\prime} \rightarrow$ $C^{1, \alpha}(B \times(-N, N))$ defined by

$$
\rho(p):=\varphi_{p}^{*} g, \quad \sigma(p):=f\left(\varphi_{p}(\cdot), \cdot\right)
$$

satisfy that

$$
\left\|\rho(p)-g_{\infty}\right\|_{C^{2, \alpha}(B)}<\delta, \quad\left\|\sigma(p)-G_{\infty}\right\|_{C^{1, \alpha}(B \times(-N, N))}<\delta .
$$

Therefore, for each $(p, \varepsilon, \bar{v})$ with $p \in M \backslash \bar{M}_{\delta}^{\prime}, \varepsilon<\delta$ and $\|\bar{v}\|_{C^{2, \alpha}\left(S^{n-1}\right)}<\delta$, it is easy to see that one can take $\left(v_{0}, \widehat{u}\right)$ depending smoothly on $p, \varepsilon$ and $\bar{v}$ : just define

$$
v_{0}(p, \varepsilon, \bar{v}):=v_{0}(\rho(p), \sigma(p), \varepsilon, \bar{v}), \quad \widehat{u}(p, \varepsilon, \bar{v})=\widehat{u}(\rho(p), \sigma(p), \varepsilon, \bar{v}) .
$$

Combining this with Proposition 3.2 applied to $\bar{M}_{\delta}^{\prime}$, and using the hypothesis on the smallness of $\left\|\phi_{p}-\phi_{\infty}\right\|_{C^{2, \alpha}\left(B_{1}\right)}$ in the first item of Assumption B to derive the smooth dependence of $\left(v_{0}, \widehat{u}\right)$ on $p \in M$, one concludes the proof.

Proposition 5.8. The claim in Proposition 3.4 also holds in the case that $M$ is an asymptotically homogeneous Riemannian manifold.

Proof. We consider the operator $\mathcal{F}$ introduced in (3.5) but, instead of being defined in an open set of $M \times[0, \infty) \times C_{\text {avg }}^{2, \alpha}\left(S^{n-1}\right)$, we define it on the open set $\mathcal{V}$ introduced in the proof of Proposition 5.7 above. We also adapt the definition of $A$, which now should be defined in $\mathcal{V}$. In particular, we have $\left(\exp _{p_{\infty}}^{h}\right)^{-1}(c(h, s, \varepsilon, \bar{v}))=$ $\varepsilon A(h, s, \varepsilon, \bar{v})$, where $c(h, s, \varepsilon, \bar{v})$ is the center of mass of $\partial B_{\varepsilon\left(1+\bar{v}+v_{0}\right)}^{h}\left(p_{\infty}\right)$.

Thus, the proof of Proposition 3.4 carries over to the new setting. We define the smooth map

$$
\overline{\mathcal{F}}(h, s, \varepsilon, \bar{v}):=(A(h, s, \varepsilon, \bar{v}), Q \mathcal{F}(h, s, \varepsilon, \bar{v})),
$$


and we apply the implicit function theorem to $\overline{\mathcal{F}}$ at the point $\left(g_{\infty}, G_{\infty}, 0,0\right)$, using Propositions 2.4 and 3.3 This implies the existence of a new $\delta>0$ such that, for each metric $h$ with $\left\|h-g_{\infty}\right\|_{C^{2, \alpha}(B)}<\delta$, each function $s$ with $\| s-$ $G_{\infty} \|_{C^{1, \alpha}(B \times(-N, N))}<\delta$, and each $\varepsilon<\delta$, there exist $\bar{v} \in C_{\text {avg }}^{2, \alpha}\left(S^{n-1}\right)$ and $a \in T_{p_{\infty}} B$, smoothly depending on $h, s$ and $\varepsilon$, such that $A(h, s, \varepsilon, \bar{v})=0$ and

$$
\mathcal{F}(h, s, \varepsilon, \bar{v})+\langle a, \cdot\rangle=0 .
$$

For such $\delta$, let $M_{\delta}$ be a relatively compact domain in $M$ that satisfies simultaneously the condition stated in Definition 5.3 and item (i) in Assumption B, and $M_{\delta}^{\prime} \supset M_{\delta}$ another relatively compact domain such that if $p \in M \backslash \bar{M}_{\delta}^{\prime}$, then $B_{R}^{g}(p) \subset M \backslash \bar{M}_{\delta}$. Again, the smooth maps $\rho$ and $\sigma$ introduced in the proof of Proposition 5.7 satisfy that

$$
\left\|\rho(p)-g_{\infty}\right\|_{C^{2, \alpha}\left(B_{R}^{g \infty}\left(p_{\infty}\right)\right)}<\delta, \quad\left\|\sigma(p)-G_{\infty}\right\|_{C^{1, \alpha}(B \times(-N, N))}<\delta .
$$

Therefore, for each $p \in M \backslash \bar{M}_{\delta}^{\prime}$ and $\varepsilon<\delta$, we can define

$$
\bar{v}_{\varepsilon, p}:=\bar{v}(\rho(p), \sigma(p), \varepsilon), \quad a_{\varepsilon, p}:=a(\rho(p), \sigma(p), \varepsilon),
$$

which depend smoothly on $p$ and $\varepsilon$, and satisfy that

$$
\mathcal{F}\left(p, \varepsilon, \bar{v}_{\varepsilon, p}\right)+\left\langle a_{\varepsilon, p}, \cdot\right\rangle=0
$$

and the center of mass of $\partial B_{\varepsilon(1+v)}^{g}(p)$ is $p$. Combining this with Proposition 3.2 applied to $\bar{M}_{\delta}^{\prime}$, one concludes the proof.

In order to conclude the adaptation of the proof of Theorem 3.1 to the asymptotically homogeneous setting, one just has to deal with the proof of Proposition 3.6. Similarly as above, the idea is to analyze separately the situation in a sufficiently large $\bar{M}_{\delta}$ and in its complement $M \backslash \bar{M}_{\delta}$. The proof of Proposition 3.6 applies directly to the former, and yields the existence of a suitable $\varepsilon_{0, \bar{M}_{\delta}}$. To deal with the latter, one just has to notice that the norms of the objects $a_{\varepsilon, p}$ and $b_{\varepsilon, p}$ in the proof of Proposition 3.6 differ from constants $a_{\varepsilon}$ and $b_{\varepsilon}$ as little as desired, by taking $\delta>0$ small enough. This yields the existence of $\varepsilon_{0, M \backslash \bar{M}_{\delta}}>0$ in the conditions of the statement of the mentioned proposition, whenever $p \in M \backslash \bar{M}_{\delta}$. Taking $\varepsilon_{0}$ as the minimum of $\varepsilon_{0, \bar{M}_{\delta}}$ and $\varepsilon_{0, M \backslash \bar{M}_{\delta}}$, we are done.

Finally, we argue how the asymptotically homogeneous assumption guarantees the existence of solutions to the overdetermined problem (1.1). Indeed, by construction, the function $\mathcal{J}_{\varepsilon}$, for each $\varepsilon<\varepsilon_{0}$, is smooth on $M$. Also by construction, $\mathcal{J}_{\varepsilon}$ is asymptotically constant, that is, there exists $c_{\varepsilon} \in \mathbb{R}$ such that for each $\delta>0$ there is a relatively compact domain $M_{\delta}$ in $M$ satisfying $\left|\mathcal{J}_{\varepsilon}(p)-c_{\varepsilon}\right|<\delta$, for all $p \in M \backslash M_{\delta}$. Then, a standard argument guarantees the existence of an extreme value for $\mathcal{J}_{\varepsilon}$ in $M$. Hence, $\mathcal{J}_{\varepsilon}$ has a critical point, which implies the existence of solutions to (1.1).

Hence we infer that if Assumption B holds, there exist $\varepsilon_{0}>0$ and a smooth function $\mathcal{J}_{\varepsilon}: M \rightarrow \mathbb{R}$ for each $\varepsilon \in\left(0, \varepsilon_{0}\right)$, such that, if $p$ is a critical point of $\mathcal{J}_{\varepsilon}$, then there exist a $C^{2, \alpha}$-domain $\Omega_{\varepsilon, p}$ containing $p$, with $\operatorname{vol}_{g}\left(\Omega_{\varepsilon, p}\right)=\left|B_{\varepsilon}\right|$, and a function $u_{\varepsilon, p} \in C^{2, \alpha}\left(\bar{\Omega}_{\varepsilon, p}\right)$, such that $u_{\varepsilon, p}$ is a positive solution to the overdetermined problem (1.1) on the domain $\Omega_{\varepsilon, p}$ with $\lambda:=\varepsilon^{-2}$. Here, $\Omega_{\varepsilon, p}$ is a $C^{2, \alpha_{\text {-small }}}$ perturbation of the geodesic ball $B_{\varepsilon}^{g}(p)$. Moreover, each function $\mathcal{J}_{\varepsilon}$ has at least a 
critical point and, hence, the existence of a family of solutions $\left(\Omega_{\varepsilon, p}, u_{\varepsilon, p}, \varepsilon^{-2}\right)$ to the overdetermined problem (1.1) for all positive $\varepsilon<\varepsilon_{0}$ is assured.

\section{Symmetry RESUlts ON MANIFOLds OF NONCONSTANT CURVATURE}

In this section we investigate overdetermined boundary problems on spaces with a high degree of symmetry (that is, with a large isometry group), but maybe not high enough to have constant curvature. We will show that the solution domains we have constructed to certain overdetermined problems inherit some symmetries from the ambient space and we will exploit this fact to prove partial symmetry results. To avoid complicated or unnatural assumptions, in this section we restrict our attention to the subclass of overdetermined problems of form (1.2), that is, with position-independent nonlinearities.

We start with a purely technical result that we will subsequently employ to derive more visual consequences. To state it in an economic way, we will sometimes write the solutions to the overdetermined boundary problem (1.2) as a triple $(u, \Omega, \lambda)$ and borrow some notation used in Step 1 of the proof of Theorem 3.1]

Proposition 6.1. Let $(M, g)$ be a compact or homogeneous Riemannian manifold and assume that the nonlinearity $G(z)$ is position-independent and satisfies Assumption A. Then:

(i) Suppose that $\left(B_{\varepsilon\left(1+v_{p}\right)}^{g}(p), u_{p}, \varepsilon^{-2}\right)$ and $\left(B_{\varepsilon\left(1+v_{q}\right)}^{g}(q), u_{q}, \varepsilon^{-2}\right)$ are solutions to the overdetermined boundary problem (1.2), with the domains $B_{\varepsilon\left(1+v_{p}\right)}^{g}(p)$ and $B_{\varepsilon\left(1+v_{q}\right)}^{g}(q)$ being centered at points $p$ and $q$ of $M$, respectively, and of the same volume: $\operatorname{vol}_{g}\left(B_{\varepsilon\left(1+v_{p}\right)}^{g}(p)\right)=\operatorname{vol}_{g}\left(B_{\varepsilon\left(1+v_{q}\right)}^{g}(q)\right)$. There is some $\delta>0$ such that, if $\varphi$ is a local isometry of $M$ with $\varphi(p)=q$ and

$$
\begin{gathered}
\varepsilon+\left\|v_{p}\right\|_{C^{2, \alpha}\left(S^{n-1}\right)}+\left\|v_{q}\right\|_{C^{2, \alpha}\left(S^{n-1}\right)}+\left\|\widehat{u}_{p}-\phi\right\|_{C^{2, \alpha}\left(B_{1}\right)}+\left\|\widehat{u}_{q}-\phi\right\|_{C^{2, \alpha}\left(B_{1}\right)}<\delta, \\
\text { then } \varphi\left(B_{\varepsilon\left(1+v_{p}\right)}^{g}(p)\right)=B_{\varepsilon\left(1+v_{q}\right)}^{g}(q) \text { and } u_{p}=u_{q} \circ \varphi .
\end{gathered}
$$

(ii) There exists $\delta>0$ such that, if $\varphi$ is an isometry of $M$ with $\varphi(p)=p$ for some $p \in M$, and $\left(B_{\varepsilon(1+v)}^{g}(p), u, \varepsilon^{-2}\right)$ is a solution to (1.2), where the domain $B_{\varepsilon(1+v)}^{g}(p)$ is centered at $p$ and

$$
\begin{array}{r}
\varepsilon+\|v\|_{C^{2, \alpha}\left(S^{n-1}\right)}+\|\widehat{u}-\phi\|_{C^{2, \alpha}\left(B_{1}\right)}<\delta, \\
\text { then } \varphi\left(B_{\varepsilon(1+v)}^{g}(p)\right)=B_{\varepsilon(1+v)}^{g}(p) \text { and } u=u \circ \varphi .
\end{array}
$$

Proof. In view of Proposition 5.1, Proposition 4.1 holds both in the compact and the homogeneous settings. Thus, let $\delta$ be as in Proposition 4.1

By the equivariance of the Laplace-Beltrami operator with respect to isometries, we have that $\left(\varphi\left(B_{\varepsilon\left(1+v_{p}\right)}^{g}(p)\right), u_{p} \circ \varphi^{-1}, \varepsilon^{-2}\right)$ is also a solution to (1.2). Notice that $\varphi^{-1}\left(B_{\varepsilon\left(1+v_{q}\right)}^{g}(q)\right)=B_{\varepsilon(1+w)}^{g}(p)$, where $w=v_{q} \circ \varphi_{* p}$. Moreover, $\varphi \circ Y_{\varepsilon, w, p}=$ $Y_{\varepsilon, v_{q}, q} \circ \varphi_{* p}$, where $Y_{\varepsilon, v, p}$ refers to the parametrization defined in (3.2). Thus, we have $\widehat{u_{q} \circ \varphi}=u_{q} \circ \varphi \circ Y_{\varepsilon, w, p}=u_{q} \circ Y_{\varepsilon, v_{q}, q} \circ \varphi_{* p}=\widehat{u}_{q} \circ \varphi_{* p}$. This, together with the facts that $\phi$ is radially symmetric and $\varphi_{* p}$ is an orthogonal transformation, implies that

$$
\left\|\widehat{u_{q} \circ \varphi}-\phi\right\|_{C^{2, \alpha}\left(B_{1}\right)}=\left\|\widehat{u}_{q}-\phi\right\|_{C^{2, \alpha}\left(B_{1}\right)}<\delta .
$$


Since moreover

$$
\|w\|_{C^{2, \alpha}\left(S^{n-1}\right)}=\left\|v_{q} \circ \varphi_{* p}\right\|_{C^{2, \alpha}\left(S^{n-1}\right)}=\left\|v_{q}\right\|_{C^{2, \alpha}\left(S^{n-1}\right)}<\delta,
$$

Proposition 4.1 applies, so it follows that

$$
\varphi^{-1}\left(B_{\left.\varepsilon\left(1+v_{q}\right)\right)}^{g}(q)\right)=B_{\varepsilon\left(1+v_{p}\right)}^{g}(p), \quad \text { and } \quad u_{q} \circ \varphi=u_{p},
$$

which proves (i).

Part (ii) follows in the same way from the previous arguments.

Part (i) of Proposition 6.1 implies in particular that, for a compact or homogeneous Riemannian manifold $M$ with isometry group $\mathbf{G}:=\operatorname{Isom}(M)$, the collection of solutions to (1.2) constructed in the proof of Theorem 3.1 from the same solution $\left(B_{1}, \phi, 1\right)$ to (1.2) are invariant under G. Part (ii) means that, if

$$
\mathbf{G}_{p}:=\{h \in \mathbf{G}: h(p)=p\}
$$

is the isotropy group at some point $p \in M$, then the solutions to (1.2) constructed in the proof of Theorem 3.1 around the point $p$ are invariant under $\mathbf{G}_{p}$. In other words, if a small perturbed ball centered at $p$ is a solution domain to (1.2) constructed as in the proof of Theorem 3.1, then such perturbed ball is invariant under $\mathbf{G}_{p}$.

Remark 6.2. If the Dirichlet problem

$$
\Delta_{\hat{g}} u+G(u)=0 \text { in } B_{1},\left.\quad u\right|_{\partial B_{1}}=0
$$

admits a unique positive solution for each metric $\hat{g}$ on $B_{1}$ that is close enough to the Euclidean one, then in Proposition 6.1 (and in the remaining results of this section) we can remove the requirement that the solutions involved are close enough to $\phi$. In this case, not only the solution domains to (1.2) constructed as in the proof of Theorem 3.1 are invariant under the isotropy group $\mathbf{G}_{p}$, but also any solution domain that is a small perturbed ball centered at $p$. Indeed, if $\left(B_{\varepsilon(1+v)}^{g}(p), u, \varepsilon^{-2}\right)$ is a solution to (1.2) and $\varepsilon$ and $\|v\|_{C^{2, \alpha}\left(S^{n-1}\right)}$ are small enough, then Proposition 3.2 guarantees the existence of a solution $\hat{u}^{\prime}$ to the Dirichlet problem for $\Delta_{\hat{g}} \hat{u}^{\prime}+G\left(\hat{u}^{\prime}\right)=$ 0 in $B_{1}$ (where $\hat{g}$ is the pullback metric of $\left.g\right|_{B_{\varepsilon(1+v)}^{g}(p)}$ under the parametrization $Y$ given in (3.2) $)$. By the uniqueness assumption in the beginning of this remark we get that $\hat{u}^{\prime}=\hat{u}$ and, as $\left\|\hat{u}^{\prime}-\phi\right\|_{C^{2, \alpha}\left(B_{1}\right)}$ is small by Proposition 3.2, then also $\|\hat{u}-\phi\|_{C^{2, \alpha}\left(B_{1}\right)}$ is small, which proves our claim.

The uniqueness for such Dirichlet problem holds, for example, for all concave functions $G$ such that $G(0)>0$, and either $G>0$ and $\lim _{t \rightarrow+\infty} G(t) t^{-1}=0$, or that there exists $\beta>0$ such that $G(\beta)=0$, see [27, §2.2]. This applies, for instance, to guarantee that small perturbations of small geodesic balls that are solution domains to the overdetermined linear problem $\Delta u+1=0$ are invariant under the isotropy. Another class of nonlinearities satisfying uniqueness of solutions is that of those nonnegative, strictly increasing and strictly convex functions $G$ with $G(0)=0$ and such that $\lim _{z \rightarrow \infty} G^{\prime}(z) \leqslant\left(\lambda_{2}\left(B_{1}\right) / \lambda_{1}\left(B_{1}\right)-\delta\right) G^{\prime}(0)$ for some positive $\delta<\lambda_{2}\left(B_{1}\right) / \lambda_{1}\left(B_{1}\right)$, see 2 (the introduction of $\delta$ with respect to the assumptions in 2] arises from the need of the uniqueness result not only for the Euclidean metric, but for all sufficiently close-by metrics on $B_{1}$ ).

Now we derive some interesting consequences of Proposition 6.1. The first one applies to the so-called two-point homogeneous spaces, which are those Riemannian manifolds $M$ such that, for any two pairs of points $p_{1}, p_{2}, q_{1}, q_{2} \in M$, there exists 
an isometry of $M$ mapping $p_{1}$ to $q_{1}$ and $p_{2}$ to $q_{2}$. It is well-known that a Riemannian manifold is two-point homogeneous if and only if it is homogeneous and isotropic, that is, for any tangent vectors $v \in T_{p} M$ and $w \in T_{q} M$, there exists an isometry $\varphi$ of $M$ such that $\varphi(p)=q$ and $\varphi_{* p} v=w$; or equivalently, for each $p \in M$ the isotropy group $\mathbf{G}_{p}$ acts transitively on the unit sphere of $T_{p} M$, via the isotropy representation $\mathbf{G}_{p} \times T_{p} M \rightarrow T_{p} M$ defined as

$$
(\varphi, v) \mapsto \varphi_{* p} v
$$

Moreover, these manifolds are Riemannian symmetric spaces [38, that is, the geodesic symmetry around each point is a global isometry [20]. Therefore, the two-point homogeneous spaces are precisely the Euclidean spaces $\mathbb{R}^{n}$, their symmetric quotients, and the rank one symmetric spaces, that is, the round spheres $S^{n}$, the real projective spaces $\mathbb{R} P^{n}$, the complex projective spaces $\mathbb{C} P^{n}$, the quaternionic projective spaces $\mathbb{H} P^{n}$, the Cayley projective plane $\mathbb{O} P^{2}$, the real hyperbolic spaces $\mathbb{R} H^{n}$, the complex hyperbolic spaces $\mathbb{C} H^{n}$, the quaternionic hyperbolic spaces $\mathbb{H} H^{n}$, and the Cayley hyperbolic plane $\mathbb{O} H^{2}$. For more information on these notions and classifications, see [3, Appendices A.3-4] and [20].

The following result asserts that, in a two-point homogeneous space with a position-independent nonlinearity, the only small perturbations of a small geodesic sphere where the overdetermined problem admits a nontrivial solution are precisely geodesic spheres, and the solution is radially symmetric (i.e. it depends only on the distance to the center). The result is a direct consequence of Propositions 4.1 and 5.1, and of the fact that, in a two-point homogeneous space, $\mathbf{G}_{p}$ acts transitively on the unit sphere of $T_{p} M$, for all $p \in M$.

Corollary 6.3. Let $M$ be a two-point homogeneous Riemannian manifold and assume that the nonlinearity $G(z)$ satisfies Assumption A. Then there exists $\delta>0$ such that, if $\left(B_{\varepsilon(1+v)}^{g}(p), u, \varepsilon^{-2}\right)$ is a solution to (1.2), where $B_{\varepsilon(1+v)}^{g}(p)$ is centered at $p$, with

$$
\varepsilon+\|v\|_{C^{2, \alpha}\left(S^{n-1}\right)}+\|\widehat{u}-\phi\|_{C^{2, \alpha}\left(B_{1}\right)}<\delta,
$$

then $B_{\varepsilon(1+v)}^{g}(p)$ is a geodesic ball and $u$ is radially symmetric around $p$.

Another interesting case occurs when the ambient manifold is a Riemannian symmetric space $M:=\mathbf{G} / \mathbf{K}$ (not necessarily of rank one), where $\mathbf{G}$ is the identity connected component of the isometry group of $M$ and $\mathbf{K}:=\mathbf{G}_{p_{0}}$ is the isotropy group at some base point $p_{0} \in M$. Here $\mathbf{G}$ acts transitively on $M$, which implies that $M$ is homogeneous. Let $k$ be the rank of $M$, that is, the dimension of the smallest totally geodesic and flat submanifold of $M$ (which is called a maximal flat of $M)$. It is a well-known fact [19, 24] that the isotropy group $\mathbf{G}_{p}$ at any point $p$ of $M=\mathbf{G} / \mathbf{K}$ acts isometrically with cohomogeneity $k$ on $M$, that is, the orbits of maximal dimension of the $\mathbf{G}_{p}$-action on $M$ have codimension $k$ in $M$. Moreover, this action is hyperpolar, which means that there exists a totally geodesic and flat submanifold $\Sigma$ of $M$ (which turns out to be a maximal flat) that intersects all the $\mathbf{G}_{p}$-orbits and always perpendicularly. In this case, the Weyl group of the action [25] is the discrete group of reflections of $\Sigma$ given by

$$
W_{\Sigma}:=N_{\mathbf{G}_{p}}(\Sigma) / Z_{\mathbf{G}_{p}}(\Sigma)
$$

where

$$
N_{\mathbf{G}_{p}}(\Sigma):=\left\{g \in \mathbf{G}_{p}: g(\Sigma)=\Sigma\right\}, \quad Z_{\mathbf{G}_{p}}(\Sigma):=\left\{g \in \mathbf{G}_{p}: g(q)=q \text { for all } q \in \Sigma\right\} .
$$


Then, it is immediate to derive the following application of Propositions 4.1 and 5.1

Corollary 6.4. Let $M$ be a symmetric space of dimension $n \geqslant 2$ and rank $k$, and let the nonlinearity $G(z)$ satisfy Assumption A. Then there exists $\delta>0$ such that, if $\left(B_{\varepsilon(1+v)}^{g}(p), u, \varepsilon^{-2}\right)$ is a solution to (1.2), where $B_{\varepsilon(1+v)}^{g}(p)$ is centered at $p$ and

$$
\varepsilon+\|v\|_{C^{2, \alpha}\left(S^{n-1}\right)}+\|\widehat{u}-\phi\|_{C^{2, \alpha}\left(B_{1}\right)}<\delta,
$$

then the solution $\left(B_{\varepsilon(1+v)}^{g}(p), u, \varepsilon^{-2}\right)$ is invariant under a group of isometries of $M$ that acts with cohomogeneity $k-1$ on the hypersurface $\partial B_{\varepsilon(1+v)}^{g}(p)$.

Moreover, let $\Sigma$ be a maximal flat through $p$. Then there exists a hypersurface $L=\partial B_{\varepsilon(1+v)}^{g}(p) \cap \Sigma$ in $\Sigma$ which is invariant under the Weyl group $W_{\Sigma}$, and such that $\partial B_{\varepsilon(1+v)}^{g}(p)$ is the union of all the $\mathbf{G}_{p}$-orbits through the points of $L$.

An interesting problem is to investigate the shape of the hypersurface $L$ of $\Sigma$, in particular, if $M$ has rank two and, hence, $L$ is a Jordan curve in $\Sigma$. A similar problem, related to the shape of soap bubbles in symmetric spaces, has been studied in [21].

Corollary 6.3 admits the following interesting improvement on harmonic spaces. Recall that a Riemannian manifold $M$ is said to be locally harmonic at $p \in M$ if all geodesic spheres of sufficiently small radius centered at $p$ have constant mean curvature. $M$ is a harmonic space if it is locally harmonic at all points. These spaces have not been classified yet, and the only known examples are the two-point homogeneous spaces mentioned above, their quotients, and certain solvable Lie groups endowed with a left-invariant metric called Damek-Ricci spaces; for more information on harmonic spaces, see [4] and [5, Chapter 6]. Notice that this result does not require the manifold to be homogeneous or compact:

Theorem 6.5. Let $M$ be a Riemannian manifold that is locally harmonic at $p \in M$ and assume that the nonlinearity $G(z)$ satisfies Assumption A. Then there exists $\delta>0$ such that, if $\left(B_{\varepsilon(1+v)}^{g}(p), u, \varepsilon^{-2}\right)$ is a solution to (1.2), where $B_{\varepsilon(1+v)}^{g}(p)$ is centered at $p$ and

$$
\varepsilon+\|v\|_{C^{2, \alpha}\left(S^{n-1}\right)}+\|\widehat{u}-\phi\|_{C^{2, \alpha}\left(B_{1}\right)}<\delta,
$$

then $B_{\varepsilon(1+v)}^{g}(p)$ is a geodesic ball and $u$ is radially symmetric around $p$.

Proof. Let $C_{0,0}^{2, \alpha}([0,1])$ denote the Banach space of functions in $C^{2, \alpha}([0,1])$ that vanish at 1 and whose first derivatives vanish at 0 . Let $\varepsilon_{0}>0$ be such that $\varepsilon_{0}\left(1+\varepsilon_{0}\right)$ is less than the injectivity radius of $M$. Consider the map

$$
\begin{aligned}
\mathcal{N}_{p}:\left[0, \varepsilon_{0}\right) \times\left(-\varepsilon_{0}, \varepsilon_{0}\right) \times C_{0,0}^{2, \alpha}([0,1]) & \rightarrow C^{0, \alpha}([0,1]) \\
(\varepsilon, \eta, \psi) & \mapsto \psi^{\prime \prime}+\left(\frac{n-1}{r}+\frac{\widehat{\theta}_{p}^{\prime}}{\hat{\theta}_{p}}\right) \psi^{\prime}+G \circ \psi .
\end{aligned}
$$

Here, $\widehat{\theta}_{p}=\theta_{p} \circ Y$, where $Y=\exp _{p}^{g} \circ T_{\varepsilon(1+\eta)}: B_{1} \subset T_{p} M \rightarrow B_{\varepsilon(1+\eta)}^{g}(p)$, and $\theta_{p}$ is the function that measures the infinitesimal change of volume around $p$, which is radial since $M$ is locally harmonic at $p$ (see 5, Chapter $6, \S \mathrm{A}-\mathrm{B}]$ ), and hence, $\widehat{\theta}_{p}$ (as well as $\psi$ ) is a function of the radial distance $r$. Notice that we can regard $\mathcal{N}_{p}$ as the operator $\Delta_{\widehat{g}}+G(\cdot)$ applied to a radial function $\psi \in C_{0,0}^{2, \alpha}\left(B_{1}\right)$, where $\widehat{g}=\varepsilon^{-2} Y^{*} g$. 
Since the function $\phi$ in Assumption A is radial, abusing the notation again, we denote also by $\phi$ the corresponding function in $C_{0,0}^{2, \alpha}([0,1])$. Then $\mathcal{N}_{p}(0,0, \phi)=0$ and $D_{\psi} \mathcal{N}_{p}(0,0, \phi)=(\cdot)^{\prime \prime}+\frac{n-1}{r}(\cdot)^{\prime}+G^{\prime} \circ \phi$. Since by Assumption A the operator $\Delta+G^{\prime} \circ \phi$ is invertible, the operator

$$
(\cdot)^{\prime \prime}+\frac{n-1}{r}(\cdot)^{\prime}+G^{\prime} \circ \phi: C_{0,0}^{2, \alpha}([0,1]) \rightarrow C^{0, \alpha}([0,1])
$$

is also invertible. Then $D_{\psi} \mathcal{N}_{p}(0,0, \phi)$ is invertible and, hence, the implicit function theorem guarantees, for each $\varepsilon$ and $\eta$ small enough, the existence of $\psi$ such that $\mathcal{N}_{p}(\varepsilon, \eta, \psi)=0$. Therefore, there exists a radial solution $\left(B_{\varepsilon(1+\eta)}^{g}(p), \psi \circ Y^{-1}, \varepsilon^{-2}\right)$ to (1.2); notice that the Neumann condition is satisfied trivially, since $\psi \circ Y^{-1}$ is radial.

Finally, let $\eta \in \mathbb{R}$ be such that $\operatorname{vol}\left(B_{\varepsilon(1+\eta)}^{g}(p)\right)=\operatorname{vol}_{g}\left(B_{\varepsilon(1+v)}^{g}(p)\right)$. Then, the application of Proposition 4.1 to the triples $\left(B_{\varepsilon(1+v)}^{g}(p), u, \varepsilon^{-2}\right)$ and $\left(B_{\varepsilon(1+\eta)}^{g}(p), \psi \circ\right.$ $\left.Y^{-1}, \varepsilon^{-2}\right)$ concludes the proof.

Observe that Theorem 6.5 combined with Remark 6.2 implies Theorem 1.4 in the Introduction.

Remark 6.6. Although the arguments developed in Section 3 do not apply directly to the first eigenvalue problem $\Delta_{g} u+\lambda_{1} u=0$ because $G(t):=t$ does not satisfy Assumption A, the symmetry results in this section apply to this case as well. The reason is that, by construction, the uniqueness result in Proposition 4.1 holds for the solutions constructed in [9, 31] to the first eigenvalue problem, just by imposing the natural requirement that the solutions are normalized to be positive and have $L^{2}$-norm equal to 1 . Since, under this additional constraint, we have uniqueness of solutions to the Dirichlet problem, Remark6.2 applies and, hence, no requirements on the proximity of $\widehat{u}$ to $\phi$ are needed.

\section{ACKNOWLEDGEMENTS}

The first author is supported by projects MTM2016-75897-P (AEI/FEDER) and ED431F 2017/03, and by the European Union's Horizon 2020 research and innovation programme under the Marie Sklodowska-Curie grant agreement No. 745722 . The second and third authors have been supported by the ERC Starting Grants 633152 and 335079, respectively. All authors acknowledge financial support from the Spanish Ministry of Economy and Competitiveness, through the Severo Ochoa Program for Centers of Excellence in R\&D (SEV-2015-0554).

\section{REFERENCES}

[1] A. D. Alexandrov: Uniqueness theorems for surfaces in the large, I, (Russian) Vestnik Leningrad Univ. Math. 11 (1956), no. 19, 5-17.

[2] H. Amann: Nonlinear eigenvalue problems having precisely two solutions, Math. Z. 150 (1976), 27-37.

[3] J. Berndt, S. Console, C. E. Olmos: Submanifolds and holonomy, CRC Press, Boca Raton, FL, 2016.

[4] J. Berndt, F. Tricerri, L. Vanhecke: Generalized Heisenberg groups and Damek-Ricci harmonic spaces, Lecture Notes in Mathematics 1598, Springer-Verlag, Berlin, 1995.

[5] A. L. Besse: Manifolds all of whose geodesics are closed, Springer-Verlag, Berlin-New York, 1978. 
[6] B. Brandolini, C. Nitsch, P. Salani, C. Trombetti: Serrin-type overdetermined problems: an alternative proof, Arch. Rational Mech. Anal. 190 (2008), no. 2, 267-280.

[7] O. Cornea, G. Lupton, J. Oprea, D. Tanré: Lusternik-Schnirelmann category, American Mathematical Society, Providence, RI, 2003.

[8] M. G. Crandall, P. H. Rabinowitz: Bifurcation, perturbation of simple eigenvalues and linearized stability, Arch. Rational Mech. Anal. 52 (1973), 161-180.

[9] E. Delay, P. Sicbaldi: Extremal domains for the first eigenvalue in a general compact Riemannian manifold, Discrete Contin. Dyn. Syst. 35 (2015), no. 12, 5799-5825.

[10] A. Enciso, D. Peralta-Salas: A symmetry result for the $p$-Laplacian in a punctured manifold, J. Math. Anal. Appl. 354 (2009), no. 2, 619-624.

[11] J. M. Espinar, A. Farina, L. Mazet: $f$-extremal domains in hyperbolic space, arXiv:1511.02659v1 [math.AP] .

[12] J. M. Espinar, J. Mao: Extremal domains on Hadamard manifolds, arXiv:1504.07453v1 [math.AP].

[13] J. M. Espinar, L. Mazet: Characterization of $f$-extremal disks, arXiv:1610.09833v2 [math.AP].

[14] L. C. Evans: Partial differential equations, American Mathematical Society, Providence, RI, 2010.

[15] M. M. Fall, I. A. Minlend: Serrin's over-determined problem on Riemannian manifolds, Adv. Calc. Var. 8 (2015), no. 4, 371-400.

[16] B. Gidas, W. M. Ni, L. Nirenberg: Symmetry and related properties via the maximum principle, Comm. Math. Phys. 68 (1979), 209-243.

[17] D. Gilbarg, N. S. Trudinger: Elliptic partial differential equations of second order, Reprint of the 1998 edition, Springer Verlag, Berlin-Heidelberg, 2001.

[18] V. Guillemin, A. Pollack: Differential topology, AMS Chelsea Publishing, Providence, RI, 2010.

[19] E. Heintze, R. Palais, C.-L. Terng, G. Thorbergsson: Hyperpolar actions and $k$-flat homogeneous spaces, J. Reine Angew. Math. 454 (1994), 163-179.

[20] S. Helgason: Differential geometry, Lie groups, and symmetric spaces, American Mathematical Society, Providence, RI, 2001.

[21] W.-T. Hsiang, W.-y. Hsiang: On the uniqueness of isoperimetric solutions and imbedded soap bubbles in non-compact symmetric spaces, I, Invent. Math. 98 (1989), 39-58.

[22] M. S. Joshi, A. Sá Barreto: Inverse scattering on asymptotically hyperbolic manifolds, Acta Math. 184 (2000), no. 1, 41-86.

[23] H. Karcher: Riemannian center of mass and mollifier smoothing, Comm. Pure Appl. Math. 30 (1977), no. 5, 509-541.

[24] A. Kollross: A classification of hyperpolar and cohomogeneity one actions, Trans. Amer. Math. Soc. 354, no. 2, 571-612.

[25] A. Kollross: Polar actions on symmetric spaces, J. Differential Geom. 77 (2007), 425-482.

[26] S. Kumaresan, J. Prajapat: Serrin's result for hyperbolic space and sphere, Duke Math. J. 91 (1998), no. 1, 17-28.

[27] P.-L. Lions: On the existence of positive solutions of semilinear elliptic equations, SIAM Rev. 24 (1982), no. 4, 441-467.

[28] F. Morabito, P. Sicbaldi: Delaunay type domains for an overdetermined elliptic problem in $\mathbb{S}^{n} \times \mathbb{R}$ and $\mathbb{H}^{n} \times \mathbb{R}$, ESAIM Control Optim. Calc. Var. 22 (2016), no. 1, 1-28.

[29] S. Nardulli: The isoperimetric profile of a smooth Riemannian manifold for small volumes, Ann. Global Anal. Geom. 36 (2009), no. 2, 111-131.

[30] M. Del Pino, F. Pacard, J. Wei: Serrin's overdetermined problem and constant mean curvature surfaces, Duke Math. J. 164 (2015), no. 14, 2643-2722.

[31] F. Pacard, P. Sicbaldi: Extremal domains for the first eigenvalue of the Laplace-Beltrami operator, Ann. Inst. Fourier 59 (2009), no. 2, 515-542.

[32] A. Ros, D. Ruiz, P. Sicbaldi: A rigidity result for overdetermined elliptic problems in the plane, Comm. Pure Appl. Math. 70 (2017), no. 7, 1223-1252.

[33] A. Ros, D. Ruiz, P. Sicbaldi: Solutions to overdetermined elliptic problems in nontrivial exterior domains, to appear in J. Eur. Math. Soc. (JEMS).

[34] F. Schlenk, P. Sicbaldi: Bifurcating extremal domains for the first eigenvalue of the Laplacian, Adv. Math. 229 (2012), no. 1, 602-632. 
[35] R. Schoen, S. T. Yau: On the proof of the positive mass conjecture in general relativity, Comm. Math. Phys. 65 (1979), no. 1, 45-76.

[36] J. Serrin: A symmetry problem in potential theory, Arch. Rational Mech. Anal. 43 (1971), 304-318.

[37] P. Sicbaldi: New extremal domains for the first eigenvalue of the Laplacian in flat tori, Calc. Var. Partial Differential Equations 37 (2010), no. 3-4, 329-344.

[38] Z. I. Szabó: A short topological proof for the symmetry of 2 point homogeneous spaces, Invent. Math. 106 (1991), no. 1, 61-64.

[39] M. Traizet: Classification of the solutions to an overdetermined elliptic problem in the plane, Geom. Funct. Anal. 24 (2014), no. 2, 690-720.

Departamento de Matemáticas, Universidad Autónoma de Madrid, and Instituto de Ciencias Matemáticas, CSIC-UAM-UC3M-UCM, Madrid, Spain.

E-mail address: miguel.dominguezv@uam.es

Instituto de Ciencias Matemáticas, Consejo Superior de Investigaciones Científicas, MADRID, SPAIN.

E-mail address: aenciso@icmat.es, dperalta@icmat.es 